\title{
Optimal Routing and Scheduling of Charge for Electric Vehicles: A Case Study
}

\author{
J. Barco, ${ }^{1,2}$ A. Guerra, ${ }^{3}$ L. Muñoz, ${ }^{3}$ and N. Quijano ${ }^{2}$ \\ ${ }^{1}$ Engineering Department, Electronics Engineering Program, Institución Universitaria CESMAG, Carrera 20A No. 14-54, \\ San Juan de Pasto, Colombia \\ ${ }^{2}$ Electrical and Electronics Engineering Department, Universidad de Los Andes, Carrera 1 Este No. 19 A 40, Bogotá, Colombia \\ ${ }^{3}$ Mechanical Engineering Department, Universidad de Los Andes, Carrera 1 Este No. 19 A 40, Bogotá, Colombia
}

Correspondence should be addressed to L. Muñoz; lui-muno@uniandes.edu.co

Received 8 April 2017; Revised 4 September 2017; Accepted 3 October 2017; Published 14 November 2017

Academic Editor: Domenico Quagliarella

Copyright (c) 2017 J. Barco et al. This is an open access article distributed under the Creative Commons Attribution License, which permits unrestricted use, distribution, and reproduction in any medium, provided the original work is properly cited.

\begin{abstract}
There are increasing interests in improving public transportation systems. One of the proposed strategies for this improvement is the use of Battery Electric Vehicles (BEVs). This approach leads to a new challenge as the BEVs' routing is exposed to the traditional routing problems of conventional vehicles, as well as the particular requirements of the electrical technologies of BEVs. Examples of BEVs' routing problems include the autonomy, battery degradation, and charge process. This work presents a differential evolution algorithm for solving an electric vehicle routing problem (EVRP). The formulation of the EVRP to be solved is based on a scheme to coordinate the BEVs' routing and recharge scheduling, considering operation and battery degradation costs. A model based on the longitudinal dynamics equation of motion estimates the energy consumption of each BEV. A case study, consisting of an airport shuttle service scenario, is used to illustrate the proposed methodology. For this transport service, the BEV energy consumption is estimated based on experimentally measured driving patterns.
\end{abstract}

\section{Introduction}

The integration of Battery Electric Vehicles (BEVs) to the public transportation system has been encouraged by the favorable efficiency in the use of energy and the reduction of $\mathrm{CO}_{2}$ emissions [1]. From the energy consumption perspective, BEVs are driven by high-efficiency motors with the possibility of implementing a regenerative braking system. Additionally, charging a BEV is less expensive than refueling a conventional vehicle because the electric energy is cheaper than its equivalent in fossil fuel (e.g., gasoline and diesel). From the emissions point of view, when a BEV is used in combination with renewable sources for the electricity generation, the outcome is a reduction in emissions associated with fossil fuel combustion; therefore, BEVs are one of the best alternatives to be integrated into cities as part of a public transportation system.

The use of BEVs in public transportation systems faces several challenges, mainly related to the combination of conventional-fuel-service characteristics with those of electric vehicles. Examples of these challenges are the routing of electric vehicles used in public transportation, the recharge scheduling, and the battery state of health $(\mathrm{SOH})$ [2]. These three challenges are treated in this work using a methodology developed for the optimal routing and scheduling of charge for BEVs.

The first challenge is the routing of BEVs. In addition to the usual routing issues, the BEVs should be routed taking particular attention to minimizing energy consumption. For the routing of BEVs, two steps are considered. The first step consists in finding the minimum consumption paths to travel between two points. In this step, it is necessary to consider the technical characteristics of BEVs. The second step consists in determining optimal routes to satisfy the transportation demand in different places and at different schedules, while minimizing energy consumption. Similar to the first step, the calculation of optimal routes is performed considering the BEVs' characteristics. For this case, the vehicle's traveling 
range is considered, mainly defined by the battery technology available [3]. This consideration could require intermediate recharge stages to extend the traveling range of the vehicles.

The second challenge is the scheduling for recharging. The recharge scheduling is a challenge as the energy rate variation during peak and valley hours must be considered. This process should be coordinated with the routing layout to guarantee a reliable operation, while the recharge costs are minimized. Additionally, for the recharge scheduling, it is necessary to consider the amount of energy required to perform the subsequent trip and the time required to recharge.

The third challenge is the $\mathrm{SOH}$ of the battery. The aim is to increase the battery lifespan to reduce the long-term operational cost. It must be considered that the useful life of the battery depends on the charge and discharge cycles [4]. This aspect is relevant because the battery is the most expensive component of the BEVs [5].

The three challenges for the implementation of BEVs for public transportation systems described in this section have been previously discussed in the literature. Nevertheless, most of those studies discuss one topic at a time. In [6, 7], the energy consumption during trips is considered. The authors propose a reduced order model for the energy consumption, which considers the BEVs' characteristics (e.g., weight, rolling resistance, and drag coefficient) and the road characteristics (e.g., grade, length, and traffic). From this model, an energy graph can be constructed to find the minimum energy consumption path between two locations using classic algorithms (e.g., Dijkstra [8] and Johnson [9]).

On the other hand, in $[7,10]$, the formulated EVRP consists of finding a set of minimum consumption routes to satisfy the demand of the users. Furthermore, some additional restrictions are included to consider the battery capacity of the BEVs. In [11], the vehicle routing problem is studied for the case of alternative fueled vehicles, considering their fueling time. In [12], a realistic energy consumption model is used for a vehicle routing problem in which the fleet includes both BEVs and diesel vehicles. In [13], the EVRP considers a heterogeneous fleet composition. In [14], an electric traveling salesman problem is studied. In [15], exact algorithms are presented for different charging conditions that generate variants of the EVRP. In $[16,17]$, the EVRP model is formulated considering the BEVs' recharge time and a set of recharge stations. Nonetheless, the battery degradation costs are not considered.

Other works such as [18-21] discuss the recharge control to minimize the energy recharge cost. Some papers $[19,21]$ study the recharge control for large fleets of BEVs. The control strategy proposed in those works minimizes the costs of energy generation and the BEVs fleet recharge. This strategy is not centralized, allowing the vehicle's autonomy preservation; nevertheless, these articles do not consider the energy consumed by each BEV nor the optimal recharge profiles obtained could increase the battery degradation.

In [22-24], another method to obtain recharge profiles for $\mathrm{BEV}$ s is discussed. In these studies, an optimization problem is formulated to minimize power losses and maximize the load factor. The formulation considers the network topology characteristics. Thereby, the obtained charge profiles contain information about the schedule and the vehicle that needs to be recharged, depending on the network connection point, whereby, the vehicles connected in the locations that induce higher losses are recharged in off-peak hours. However, these studies do not consider the effect of the recharge profile on the battery life nor the recharge cost in the objective function.

In $[25,26]$, the battery SOH is studied through a degradation model for the lithium-ion batteries. The model considers the main features that influence battery degradation such as the mean state of charge (SOC), depth of discharge (DOD), and battery temperature. In [25], some models to minimize the battery degradation and the cost of energy recharge are proposed. The recharge profile obtained is considerably different to those obtained in $[19,21]$, where it is calculated considering only the recharge cost.

In $[27,28]$, the EVRP is combined with infrastructure problems. In [27], the combination of the EVRP and the location-routing problem is presented, including the determination of battery swap stations and also the possibility of intermediate stops. In [28], different scenarios for the use of artificial intelligence for the management of BEVs are presented; those scenarios include issues for the grid-tovehicle interaction as load balancing, energy pricing, placing of recharging places, and rerouting of BEVs to charge points.

This work proposes a methodology that relates the three challenges presented before to determine: minimum consumption paths, a set of optimal routes, the route assignment for each vehicle, and the recharge scheduling for electric vehicles for public transportation. A centralized controller based on a program that minimizes the costs of electric vehicles' operation is proposed. The objective function to minimize considers both the recharge cost and the cost associated with the battery degradation caused by route assignation and recharge cycles.

As a case study, an airport shuttle service attended by a fleet of BEVs is used to analyze the EVRP, while considering the topics exposed previously. This service consists of transporting passengers from a hotel to a nearby airport. Comparative results between the recharge scheduling obtained with a reduced order model and real energy consumption data of an electric vehicle are presented. Finally, findings on the BEVs' charge patterns, routing, and operational costs are presented. This information could be useful for public transportation companies interested in upgrading their fleet to BEVs.

This paper is organized as follows. Section 2 presents the energy consumption and optimal routing model; the energy consumption of the BEVs is estimated using a model based on the longitudinal dynamics equation of motion. Section 3 introduces the recharge scheduling problem, comprised of a route assignation and the charge model. Section 4 describes the battery degradation model. Section 5 presents an evolutionary algorithm to solve the proposed EVRP. Section 6 describes the case study and presents simulation results. Section 7 presents the conclusion.

\section{Optimal Routing for BEVs}

The considered scenario takes into account the presence of an operation center (OC), which coordinates both the transport 


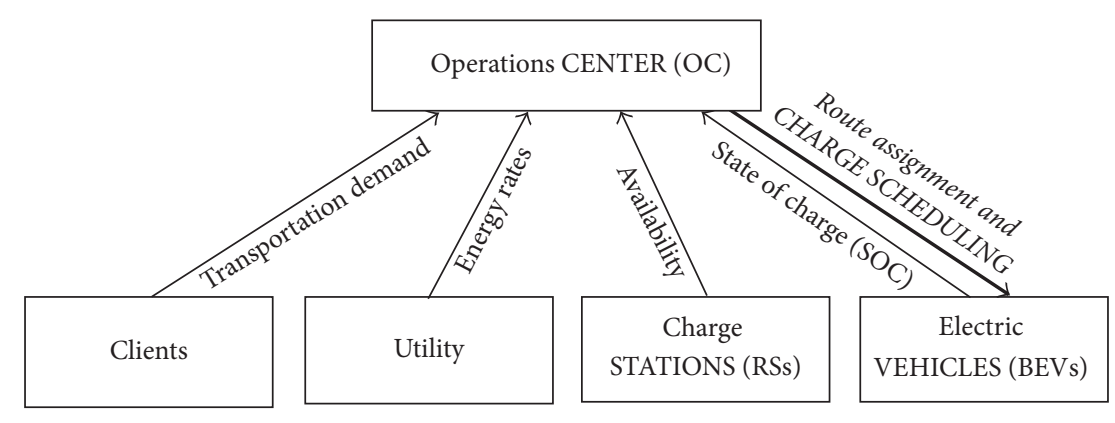

Figure 1: Operation center scheme.

service and the charge scheduling. The transport service consists of a BEV fleet that offers an airport shuttle service.

The charge scheduling seeks to minimize the cost; for this reason, the OC assigns routes to the BEVs that satisfy the transportation demand and establishes the charge schedules in the available charge stations (RSs) according to the energy rate. To coordinate the operation of the BEV fleet, the OC can communicate with BEVs, clients (i.e., potential passengers), RSs, and the public utility as presented in Figure 1. In this way, the OC receives information regarding requests from customers one day in advance, availability of RSs, state of charge of BEVs, and energy rates from the public utility. The route assignment and the charge scheduling are calculated and transmitted to the electric vehicles.

In the next subsections, the models of the systems, in which the OC is based, are described. These models are presented in the following order: estimation of energy consumption on the road, determination of optimal routes, assignment of routes, scheduling of a specific charge problem, and the solution method based on differential evolution.

2.1. Energy Consumption on the Road. For a BEV, the energy consumption on the road is sensitive to environment, road, and vehicle characteristics. Factors as the road grade and the travel speed can significantly influence the energy consumption. Additionally, the traffic conditions and other environmental factors can cause acceleration/deceleration rates that have an impact on the energy consumption of the vehicle. Consequently, a model that considers a vehicle moving at a constant speed is not sufficient.

The energy consumption model presented in this section is based on the longitudinal dynamics equations of motion presented in [29]. This model allows determining the instantaneous power consumption of the vehicle during its operation, considering the resistive loads imposed on the vehicle (i.e., aerodynamic drag force, rolling resistance force due to contact between tires and road, and longitudinal component of the gravitational force due to driving on hilly roads). For the implementation, the rolling resistance force was modeled by considering a constant rolling coefficient; hence, the rolling resistance force changes linearly with the changes of the normal force between the vehicle and the road [29].

The energy consumption of the vehicle is determined by integrating the instantaneous power consumption of the

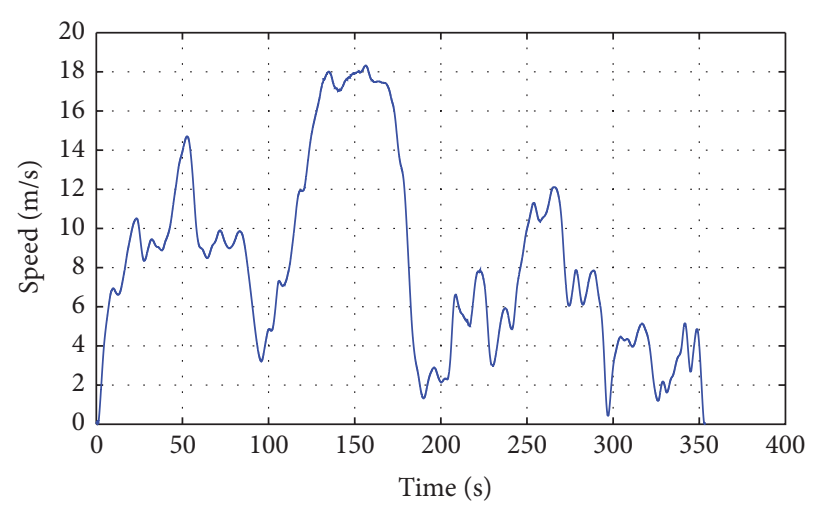

Figure 2: Example of a speed profile for a route.

vehicle, which is a function of the environment, road, and vehicle characteristics. For the total energy computation, the energy consumed by a BEV while accelerating or in motion with a constant speed is considered; the energy recovered by the system with regenerative braking is not considered.

The road characteristics are modeled in a directed graph $\mathscr{G}=(\mathscr{V}, \mathscr{E})$. The vertices (i.e., key points or nodes) $v \in \mathscr{V}$ represent the points of special interest on the street maps. The edges $\varepsilon_{i j} \in \mathscr{E}$, where $\varepsilon_{i j}=\left(v_{i}, v_{j}\right)$, represent road sections between key points. The model assumes that, for each edge, the representative driving pattern associated with the given edge is known and it is represented by its speed profile (see Figure 2). Hence, there is a function $s: \mathscr{E} \rightarrow \kappa(\mathbb{R})$, where $s_{i j}=s\left(\varepsilon_{i j}\right)$ corresponds to the representative speed profile associated with the road section that connects the vertices $v_{i}$ and $v_{j}$, where $\kappa(\mathbb{R})$ is the space of continuous real functions with compact support.

The model also assumes that the key point elevation $z$ : $\mathscr{V} \rightarrow \mathbb{R}$ is known. On the other hand, (1) presents the terms involved into the power consumption of the vehicle:

$$
\begin{aligned}
& P_{i, j}(t) \\
& =\frac{P_{\text {aerodynamic }}(t)+P_{\text {rolling }}(t)+P_{\text {specific }}(t)+P_{\text {gravitational }}(t)}{\widehat{\eta}} .
\end{aligned}
$$

The first term on the right side of (1) corresponds to the power dissipated by aerodynamic drag. The second term corresponds to the rolling resistance between tires and asphalt. The third term stands for the specific power required to overcome 
the vehicle inertia, and the fourth term corresponds to the power consumed/gained due to the height of the vehicle originated by the road grade, represented by angle $\gamma$. The vehicle characteristics that influence the power consumption are mass $(m)$, frontal area $(A)$, drag and rolling resistance coefficients $\left(C_{D}, f_{r}\right)$, and the powertrain efficiency $(\widehat{\eta})$. Given these characteristics, the power consumption at instant $t$ of the trip between vertex $i$ and vertex $j$ is given by

$$
P_{i, j}(t)=\frac{(1 / 2) \rho A C_{D}\left[s_{i j}(t)\right]^{3}+m g f_{r} s_{i j}(t)+m\left(d s_{i j} / d t\right)(t) s_{i j}(t)+m g \tan (\gamma) s_{i j}(t)}{\widehat{\eta}} .
$$

The energy consumption can be calculated by integrating (2) over the total time of each displacement between vertices, to obtain the energy consumption associated with the trip.
Under the assumption of constant powertrain efficiency, the gravitational component can be analytically integrated. This leads to the following expression:

$$
c_{i, j}=\int_{0}^{t_{f}}\left(\frac{(1 / 2) \rho A C_{D}\left[s_{i j}(t)\right]^{3}+m g f_{r} s_{i j}(t)+m\left(d s_{i j} / d t\right)(t) s_{i j}(t)}{\hat{\eta}}\right) d t-\frac{m g\left[z\left(v_{j}\right)-z\left(v_{i}\right)\right]}{\hat{\eta}} .
$$

Finally, energy consumption can be computed for an arbitrary path composed of different edges of the graph. A path $P_{h}$ is defined as a sequence of $l$ vertices $\left(v_{1}, v_{2}, \ldots, v_{l}\right)$ with $\left(v_{i}, v_{i+1}\right) \in \mathscr{E}$ for $i=1, \ldots, l-1$. The energy necessary to travel along the path $P_{h}$ in the road network is the sum of the energy consumed to complete each one of the road sections that compose the path as follows:

$$
C\left(P_{h}\right)=\sum_{i=1}^{l-1} c_{i, j}
$$

2.2. Determination of Routes. Similar to the classical vehicle routing problem formulated in [30], an EVRP is proposed to model the airport shuttle service. It consists of searching a set of minimum consumption routes that satisfy the transportation demand and the operational constraints. Figure 1 presents the symbols used to explain the routing model.

The EVRP model is formulated on the energy graph $\mathscr{G}_{s}=$ $\left(\mathscr{V}_{s}, \mathscr{C}_{s}\right)$, which is a simplification of the road network. The graph nodes are composed of $\mathscr{V}_{s}=\left\{v_{d_{1}}, v_{d_{2}}\right\} \cup C \cup R_{s}$, where $C$ is the set of nodes within a transportation demand, and $R_{s}$ is the set of RSs. The expression $2 \cdot|C|$ is the number of requests made by customers, where the operator $|\cdot|$ represents the set module. The depot nodes are denoted by $v_{d_{1}}$ and $v_{d_{2}}$, which represent the route's starting and ending nodes. The graph edges, defined by $\mathscr{E}_{s}=\left\{\left(v_{i}, v_{j}\right) \mid v_{i}, v_{j} \in \mathscr{V}_{s}\right\}$, where $\left(v_{i}, v_{j}\right) \in \mathscr{E}_{s}$, are paths of minimum consumption that can be found through routing algorithms applied to the road network model. Therefore, the transportation demand implies $m$ requests to pick up passengers and $m$ requests to drop off passengers, each one with its associated number of passengers. The requests are identified by two nodes $i$ and $m+i$, corresponding to the pick-up and drop-off stops, respectively. The set of pick-up nodes is denoted by $P=$ $\{1, \ldots, m\}$, and the set of drop-off nodes is denoted by $D=$ $\{m+1, \ldots, 2 m\}$. Therefore, it is possible to define $C=P \cup D$ and if the request $i$ consists of transporting $q_{i}$ passengers from $i$ to $m+i$, then $q_{(m+i)}=-q_{i}$.

The energy consumed during the trip from $i$ to $j$ is given by $c_{i j}$, and the traveling time is $t_{i j}$, where $i, j \in \mathscr{V}_{s}$. Each customer $i \in C$ has a time window $\left[a_{i}, b_{i}\right]$ in which the service must take place. Electric vehicles have a maximum passenger capacity $Q$ and a maximum battery capacity $B$. The objective function minimizes the energy consumption for all routes $H$, meaning

$$
\min \sum_{h \in H} \sum_{(i, j) \in \mathscr{E}_{s}} c_{i j} x_{i j}^{h}
$$

where $h \in H$ is the set of routes to satisfy all the transportation demand and $x_{i j}^{h}$ are the flow variables, which are equal to 1 if the $\operatorname{arc}(i, j)$ is used on route $h$, or 0 otherwise. In addition, two constraints are defined to guarantee that all the passenger demands are satisfied, which are

$$
\begin{aligned}
& \sum_{h \in H} \sum_{j \in \mathscr{V}_{s} \backslash v_{d_{1}}} x_{i j}^{h}=1 \quad \forall i \in P, \\
& \sum_{i \in \mathscr{V}_{s} \backslash v_{d_{2}}} x_{i j}^{h}-\sum_{i \in \mathscr{V}_{s} \backslash v_{d_{1}}} x_{j, m+1}^{h}=0 \\
& \forall h \in H, j \in \mathscr{V}_{s} \backslash\left\{v_{d_{1}}, v_{d_{2}}\right\} .
\end{aligned}
$$

Equation (6) guarantees that each node with passenger demand is attended or visited by one route. Moreover, (7) forces passengers picked up at node $i$ to be dropped off at node $m+i$. Three constraints are also defined to satisfy the flow through vertices; these are

$$
\begin{aligned}
& \sum_{i \in \mathscr{V}_{s} \backslash v_{d_{2}}} x_{i j}^{h}-\sum_{i \in \mathscr{V}_{s} \backslash v_{d_{1}}} x_{j i}^{h}=0 \\
& \forall h \in H, j \in \mathscr{V}_{s} \backslash\left\{v_{d_{1}}, v_{d_{2}}\right\}, i \neq j,
\end{aligned}
$$




$$
\begin{gathered}
\sum_{j \in \mathscr{V} s \backslash v_{d_{2}}} x_{v_{d_{1}}, j}^{h} \leq 1, \quad \forall h \in H, \\
\sum_{i \in \mathscr{V} s \backslash v_{d_{2}}} x_{i, v_{d_{2}}}^{h} \leq 1, \quad \forall h \in H .
\end{gathered}
$$

Equation (8) indicates that if a route $h$ arrives at node $j$, then the same route must depart from node $j$, keeping the flow equilibrium. Also, (9) indicates that a route $h$ can exit one time of the departing node $v_{d_{1}}$. On the other hand, (10) indicates that route $h$ can enter once to the arriving node $v_{d_{2}}$. Furthermore, three sets of constraints are defined: (i) time constraints; (ii) capacity constraints; and (iii) energy constraints. The capacity constraints are

$$
\begin{aligned}
& y_{j}^{h} \geq y_{i}^{h}+q_{j}-Q+Q x_{i j}^{h}, \quad \forall h \in H, i, j \in P, i \neq j, \\
& q_{j} \leq y_{j}^{h} \leq Q, \quad \forall h \in H, \quad j \in \mathscr{V}_{s}, \\
& 0 \leq y_{j+m}^{h} \leq Q-q_{j}, \quad \forall h \in H, \quad j \in P,
\end{aligned}
$$

where $y_{j}^{h}$ is the capacity variable representing the amount of passengers picked up or dropped off during a trip along route $h$ to node $j$. Therefore, (11) counts the number of passengers traveling along route $h$, with constraints (12) and (13) guaranteeing that the capacity variable does not exceed the maximum capacity $Q$. The time constraints are

$$
\begin{aligned}
& w_{j}^{h} \geq w_{i}^{h}+t_{i j}-M+M x_{i j}^{h}, \\
& \qquad h \in H, i, j \in \mathscr{V}_{s}, i \neq j, \\
& a_{j} \leq w_{j}^{h} \leq b_{j}, \quad \forall h \in H, j \in \mathscr{V}_{s},
\end{aligned}
$$

where $w_{i}^{h}$ is the time variable specifying the instant at which passenger $i$ is picked up or dropped off and $M$ is a constant higher than any value of $w_{i}^{h}$. Therefore, (14) counts the elapsed time until passenger $i$ is attended by route $h$. In addition, the constraint in (15) guarantees that passenger $i$ is picked up or dropped off within the time window. Finally, the energy constraints are

$$
\begin{aligned}
& e_{j}^{h} \leq e_{i}^{h}-c_{i j} x_{i j}^{h}+B-B x_{i j}^{h}, \\
& \qquad h \in H, i, j \in \mathscr{V}_{s}, i \neq j, \\
& e_{\min } \leq e_{j}^{h}, \quad \forall h \in H, \quad j \in \mathscr{V}_{s},
\end{aligned}
$$

where $e_{i}^{h}$ is the energy variable specifying the remaining battery charge level when route $h$ arrives to vertex $i$. Additionally, (16) allows computing the battery level based on the traveled vertices sequence. Moreover, (17) guarantees that at the end of route $h$ the battery level will never drop below the minimum level $e_{\min }$.

The solution of proposed EVPR model is a set of optimal routes, $H$, which minimizes the energy consumed while satisfying the passenger demand, indirectly reducing the charge costs.

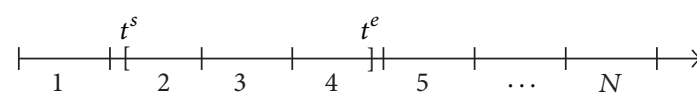

FIgURE 3: Timeline in the programming horizon.

\section{Scheduling of Charge Problem}

In this section, the scheduling of charge for BEVs is presented. First, the assignment of routes for the BEV fleet is defined. Then, the model for the charge control is established. Finally, the problem formulation is presented.

3.1. Assignment of Routes. The assignment of routes consists of the distribution of all the optimal routes $S$ within the BEV fleet. It is considered that the assignment of a set of routes in the vehicle fleet, $K$, is performed in a programming horizon $N$. The programming horizon is illustrated in Figure 3.

The assignment of one route to a specific BEV is denoted by $a_{s}^{k}$, with $k \in K$, a binary variable, which is equal to 1 if route $s$ is assigned to an $\mathrm{EV}$, or 0 otherwise. A binary variable for "unavailability" is defined. This variable indicates that some periods of $i \in N$ are used to travel along route s assigned to a $\mathrm{BEV}$. The unavailability variable is defined as

$$
\begin{gathered}
d_{s}^{k}(i)= \begin{cases}1, & \text { if } t_{s}^{s} \leq i \leq t_{s}^{e}, \\
0, & \text { otherwise },\end{cases} \\
d_{s 1}^{k}(i)+d_{s 2}^{k}(i) \leq 1, \quad \forall i \in N, \quad \forall k \in K, s_{1}, s_{2} \in S,
\end{gathered}
$$

where $\left[t_{s}^{s}, t_{e}^{s}\right]$ is the interval of duration of each route $s$, with starting time $t_{s}^{s}$ and ending time $t_{e}^{s}$. This way, variable $d_{s}^{k}(i)$ allows locating each route $s$ on the horizon $N$, while (19) guarantees that two different routes $\left(s_{i}, s_{2}\right)$, with similar duration intervals, are not assigned at the same time to the same BEV. On the other hand, the energy consumed by the BEV $k$ in route $s$ for interval $i$ of the horizon $N$ is defined as $e_{s}^{k}(i)$.

Finally, an assignation profile is defined with vector $\aleph=$ $\left\{a_{s}^{k}: \sum_{k \in K} \sum_{s \in S} a_{s}^{k}=|S|\right\}$, where the double sum guarantees that all the routes of $S$ are assigned to the electric vehicles composing the fleet. Considering this, an assignation profile is valid if the constraint on (19) is satisfied for all the BEVs in the fleet.

3.2. Model for Charge Control. A charge control is proposed for a BEV fleet $K$, over a programming horizon $N$, based on the work presented in [19]. The SOC is defined for each vehicle $k \in K$ at instant $i \in N$ as $\operatorname{SOC}^{k}(i)$, with the following considerations:

$$
\begin{aligned}
\operatorname{SOC}^{k}(i)= & \operatorname{SOC}^{k}(i-1) \\
& +\sum_{z \in R}\left[\eta_{z} r_{z} u_{z}^{k}(i)-\eta_{z}^{d} r_{z}^{d} v_{z}^{k}(i)-e_{z}^{k}(i)\right] \\
& -\sum_{s \in S} e_{s}^{k}(i), \quad \forall i \in N, \forall k \in K,
\end{aligned}
$$


where $u_{z}^{k}(i)$ is a binary variable, equal to 1 when the vehicle $k$ performs a charge action at instant $i, r_{z}$ is the charge rate, and $\eta_{z}$ is the efficiency of the charge process; $v_{z}^{k}(i)$ is a binary variable, which allows us to integrate the vehicle to building (V2B) energy discharge action, this variable is equal to 1 when the vehicle $k$ performs a discharge action at instant $i, r_{z}^{d}$ is the discharge rate, and $\eta_{z}^{d}$ is the efficiency of the discharge process. Subscript $z \in R$ indicates the place where the charge or discharge is performed. Variable $e_{z}^{k}(i)$ indicates the energy consumed during the displacement towards a $\mathrm{RS} z$, explained in the next section. Also, a restriction for SOC of the vehicles must be considered as

$$
\mathrm{SOC}_{\text {min }} \leq \operatorname{SOC}^{k}(i) \leq \mathrm{SOC}_{\text {max }} \quad i \in N, \forall k \in K,
$$

where $\mathrm{SOC}_{\min }$ and $\mathrm{SOC}_{\max }$ are the lower and upper bounds of SOC, respectively. Both bounds can be established according to the battery manufacturer recommendation, considering the battery technology. For this study, the initial and final $\mathrm{SOC}$ are equal to the $\mathrm{SOC}_{\max }$ value to guarantee that the vehicle begins and ends in the programming horizon with the same battery energy level, meaning

$$
\begin{aligned}
\operatorname{SOC}^{k}(0) & =\operatorname{SOC}_{\text {max }}, \\
\operatorname{SOC}^{k}(N) & =\mathrm{SOC}_{\text {max }},
\end{aligned}
$$

\section{$\forall k \in K$,}

where $\operatorname{SOC}^{k}(0)$ and $\operatorname{SOC}^{k}(N)$ are the initial and final states of charge of a BEV $k$. Finally, a charge profile for a vehicle $k$ is defined as a set of charge actions $u_{z}^{k}(i)$. A charge profile for the BEV $k$ is denoted $\mathbf{u}^{k}$ and defined as $\mathbf{u}^{k}=\left\{u_{z}^{k}(i)\right.$ : $\left.\eta_{z} r_{z} \sum_{i \in N} u_{z}^{k}(i)=\sum_{i \in N}\left(e_{s}^{k}(i)+e_{z}^{k}(i)\right)\right\}$, where the sum of all the charge actions must be equal to the energy consumed during all the trips. The profile $\mathbf{u}^{k}$ is valid if the constraints on (20) to (22) are satisfied.

3.3. Reroute of Charge Stations. In the model for determining routes, it is assumed that when a vehicle $k$ is not traveling along any of them, then the vehicle is parked on the departing/arriving node $\left(v_{d_{1}}, v_{d_{2}}\right)$, where the vehicles can be charged in a private station denoted by $R_{1} \in R$. It is also considered that the vehicle can be charged in public stations identified in the operation zones; these charge actions on public stations might be necessary when the vehicles do not have enough energy to return to the arriving/departing node or when the private stations are occupied by other vehicles.

When considering that the vehicles can be charged in public stations, it must also be considered that their trajectory has to be reprogrammed or their initially assigned route must be modified, which is known as rerouting. Rerouting is defined as the redirection of the vehicle towards a station to perform a charge action $u_{z}^{k}: z \neq R_{1}$ without altering the vehicle's destination. The redirecting or rerouting is denoted with subscript $z$ in the charge action $u_{z}^{k}$. If subscript $z$ indicates a place different than $R_{1}$, then BEV $k$ must travel to another station $R_{2} \in R$, to perform the charge action.
It must be considered that redirecting towards a station implies that the BEV must have available time to travel back and forth to the RS. Similarly, the BEV must have some time intervals available to charge the energy that it needs; this is assured using two constraints. The first constraint is

$$
\begin{aligned}
& d_{s}^{k}(j)+u_{y}^{k}(j)=0 \\
& \quad i_{z}-\Delta i_{z} \leq j<i_{z}, \forall y \neq z, y \in R,
\end{aligned}
$$

where $i_{z} \in N$ is the interval at the beginning of the charge and $u_{z}^{k}, \Delta i_{z}$ is the number of time intervals that a BEV $k$ needs to travel from $v_{d_{1}}$ to $z$. If the result of (23) is zero, the BEV $k$ can be rerouted towards a public recharge station because it has enough time to perform the action. The second constraint is

$$
\begin{aligned}
& d_{s}^{k}(j)+u_{y}^{k}(j)=0 \\
& i_{z}+\Delta i_{z} \leq j<i_{z}+\Delta i_{u_{z}}+\Delta i_{v_{d_{1}}}, \forall y \neq z,
\end{aligned}
$$

where $\Delta i_{u_{z}}$ is the number of time intervals in which the charge action needs to be completed, and $\Delta i_{v_{d_{1}}}$ is the number of time intervals that a BEV $k$ needs to travel from $z$ to $v_{d_{1}}$. Equations (23) and (24) guarantee that BEV $k$ can be rerouted when there is enough time available. On the other hand, the energy consumed in the rerouted trip is denoted as $e_{z}^{k}$, which corresponds to the energy consumed traveling from $v_{d_{1}}$ to $z$ and back. This is considered in (20) assuring that the vehicle has the energy necessary to perform the displacement needed when redirected.

3.4. Optimization Problem of Charge for BEVs. The proposed optimization problem seeks to minimize the operation cost of the electric vehicle fleet, defined as

$$
\min \sum_{z \in R} \sum_{k \in K} \sum_{i \in N} p_{z}(i) u_{z}^{k}(i)+\sum_{k \in K} c_{\mathrm{deg}}^{k}
$$

The first term is the charge energy cost for all the BEVs in the fleet, where $p_{x}(i)$ is the energy rate in the recharge station $x$ at instant $i$; the last term of the objective function is the cost of battery degradation for all the BEVs in the fleet. The objective function in (25) is subjected to a valid assignment profile $\boldsymbol{N}$ and a valid charge profile $\mathbf{u}^{k}$ for all the BEVs $k \in K$. Besides, if there is redirecting towards public stations, then the constraints in (23) and (24) should be satisfied.

Likewise, a constraint related to the recharge stations' availability is defined as $\sum_{k \in K} u_{z}^{k}(i) \leq A_{z}(i), \forall i \in N, z \in$ $R$ to guarantee that the charge actions are programmed in available charge points, where $A_{z}(i)$ is the number of spaces available in recharge station $z$.

\section{Battery Degradation Model}

In this section, a simplified version of the lithium-ion battery degradation model proposed in [25] is presented.

The model used in this work estimates the cost of battery degradation $c_{\mathrm{deg}}$ in terms of the reduction of the lifespan of a battery, which is a function of three factors: temperature, 
SOC, and DOD. These factors are related to the cost as follows:

$$
c_{\mathrm{deg}}=c_{\mathrm{bat}}\left(L_{\mathrm{Q}, T}+L_{\mathrm{Q}, \mathrm{SOC}}+L_{\mathrm{Q}, \mathrm{DOD}}\right) \text {, }
$$

where $c_{\text {bat }}$ is the initial cost of the battery and $L_{\mathrm{Q}, T}, L_{\mathrm{Q}, \mathrm{SOC}}$, and $L_{\mathrm{Q}, \mathrm{DOD}}$ are the percentage terms of battery degradation due to temperature, SOC, and DOD, respectively.

Each of the terms $L_{\mathrm{Q}, T}, L_{\mathrm{Q}, \mathrm{SOC}}$, and $L_{\mathrm{Q}, \mathrm{DOD}}$ can be interpreted as a ratio $\left(\Delta L_{x} / L_{x}\right)$, where $\Delta L_{x}$ is the battery lifetime degradation due to a complete charge cycle during a day, and $L_{X}$ is the total lifetime of the battery if the evaluated charge cycle is repeated until the end of the battery life, that is, when the energy capacity of the battery is lower than $80 \%$.

The term $L_{Q, T}$ refers to the battery degradation due to temperature and charge time. This term is proportional to the charge power since a high operation temperature corresponds to a high charge power. This term is defined as

$$
\begin{aligned}
L_{\mathrm{Q}, T}= & \int_{t_{\mathrm{ch}}} \frac{1}{n_{h y} l_{y}\left(T_{\mathrm{amb}}+R_{\mathrm{th}}\left|P_{t}(t)\right|\right)} d t \\
& +\frac{t_{\mathrm{max}}-t_{\mathrm{ch}}}{n_{h y} l\left(T_{\mathrm{amb}}\right)},
\end{aligned}
$$

where $t_{\mathrm{ch}}$ is the charge time in hours, $t_{\max }$ is the maximum time available to perform a charge in hours, and $n_{h y}$ is the number of hours in a year. $T_{\mathrm{amb}}$ is the ambient temperature, established with a constant value of $25^{\circ} \mathrm{C}$, and $R_{\text {th }}$ is the thermal resistance of the battery, established with a constant value of $2^{\circ} \mathrm{C} / \mathrm{kW}$. $P_{t}$ corresponds to the charge or discharge power in $(\mathrm{kW})$, and $l_{y}(T)$ is a function that estimates the total number of years that the battery will last at a temperature $T$, defined as $l_{y}(T)=a e^{b / T}$, where $a$ and $b$ are the parameters of the model of a lithium battery fixed as $\mathbf{a}=3.73 \times 10^{-4}$ and $\mathbf{b}=636$ according to [25].

The term $L_{\mathrm{Q}, \mathrm{SOC}}$ describes the battery lifetime degradation due to average SOC, defined as

$$
L_{\mathrm{Q}, \mathrm{SOC}}=\frac{m_{a} \mathrm{SOC}_{\mathrm{avg}}-d_{a}}{\mathrm{CF}_{\max } y_{p} n_{h y}},
$$

where $m_{a}=1.6 \times 10^{-5}$ and $d_{a}=6.4 \times 10^{-6}$ are the parameters of the model for the lithium-ion batteries defined in [25], while $y_{p}=15$ corresponds to the number of years which the battery is expected to last, and $\mathrm{CF}_{\max }=0.80$ represents the fading capacity at the end of the battery life. From (28), it can be noticed that $L_{\mathrm{Q}, \mathrm{SOC}}$ is proportional to $\mathrm{SOC}_{\mathrm{avg}}$; thus, a high $\mathrm{SOC}_{\mathrm{avg}}$ reduces the useful life of the battery more than a moderated one.

The term $L_{\mathrm{Q}, \mathrm{DOD}}$ describes the degradation of the battery lifetime due to the mean DOD. This term is calculated using the energy performance concept [31]; hence, $L_{\mathrm{Q}, \mathrm{DOD}}$ is the ratio between the energy throughput used in a complete charge cycle and the energy throughput used during the battery lifetime, defined as

$$
L_{\mathrm{Q}, \mathrm{DOD}}=\frac{B \sum_{i=1}^{N_{c}} \mathrm{DOD}_{i}}{N_{l}\left(\mathrm{DOD}_{\mathrm{avg}}\right) * \mathrm{DOD}_{\mathrm{avg}} * B},
$$

where $\mathrm{DOD}_{i}$ is the $i$ th subcycle in a complete charge cycle, $N_{c}$ is the number of subcycles, DOD ${ }_{\text {avg }}$ is the average DOD cycle, and $N_{l}$ (DOD) is the life time of the battery in cycles for a given DOD. This is defined as $N_{l}$ (DOD) $=(\mathrm{DOD} /$ $145.71)^{-1 / 0.6844}$, which is fitted for lithium-ion battery technology.

Summarizing, the model has two attributes that allow its integration to the optimization problem. First, it allows computing the battery degradation cost of a BEV due to the actions of charge and discharge previously programmed. Second, the simplicity of the model allows performing the calculations in a fast way, reducing the use of computational resources, embedding it with the optimization problem and its solution method, explained in the next section.

\section{Differential Evolution}

A differential evolution (DE) algorithm is presented to solve the problem formulated in previous sections. The $\mathrm{DE}$ is a direct stochastic search algorithm based on the evolution of a population, which was developed by Storn et al. in 1996 $[32,33]$. The DE operates through steps that are similar to those used in a standard evolutionary algorithm (EA).

The DE employs the differences between members of the population to explore the objective function. For this reason, it does not use a probability function to generate the offspring. Hence, the DE uses an approach that is less stochastic, making it more efficient for solving several kinds of problems [34].

In the next subsections, the format of a solution and the steps composing the DE algorithm are presented.

5.1. Format of a Solution. Before solving the problem, a possible solution must be represented in a format that the DE can optimize. For this case, the possible solution of the electric vehicle charge problem is represented by the valid assignment profile $\aleph$, and the collection of the charge profiles $\mathbf{u}^{k}$, associated with each vehicle. The assignment profile contains each route assignation variable $a_{k}^{h}, \forall k \in K, h \in H$. For each vehicle $k \in K$, the associated charge profile contains each charge action variable for that vehicle, $u_{z}^{k}(n), \forall z \in R$, $n \in N$.

$$
\begin{aligned}
X^{u} & =\left\{u_{1}^{1}(1), \ldots, u_{1}^{1}(N), \ldots, u_{1}^{|K|}(1), \ldots, u_{1}^{|K|}(N), \ldots,\right. \\
& \left.u_{|R|}^{1}(1), \ldots, u_{|R|}^{1}(N), \ldots, u_{|R|}^{|K|}(1), \ldots, u_{|R|}^{|K|}(N)\right\} \\
X^{a} & =\left\{a_{1}^{1}, \ldots, a_{1}^{|K|}, \ldots, a_{|H|}^{1}, \ldots, a_{|H|}^{|K|}\right\}
\end{aligned}
$$

where $X^{u}$ and $X^{a}$, maintaining the notation of [32], are denoted as vectors of charge and assignment parameters, respectively. Considering this, $X=X^{u} \cup X^{a}$ is defined as a feasible solution for the electric vehicle charge problem, denoted as the parameter vector $X$ in the development of each step of the DE algorithm, which are described next.

5.2. Population Initialization. Similar to the EA, the DE steps are (i) population initialization; (ii) mutation; (iii) crossing over; and (iv) selection. A population is composed of a number $N_{p}$ of vectors $X$, where each vector is known as an 
individual. The following notation is adopted to represent a vector of parameters $i \in N_{P}$ of the population in the current generation $G_{e}$

$$
X_{i, G_{e}}=\left[x_{1, i, G_{e}}, x_{2, i, G_{e}}, \ldots, x_{m_{v}, i, G_{e}}\right]
$$

where $G_{e}=0,1, \ldots, G_{\max }$ denotes the future generations, $G_{\max }$ the last generation, and $m_{v}$ the size or dimension of the parameter vector.

The population of vectors is produced through a random generator, where each vector must meet the constraints; otherwise, it will be rejected and will not be part of the population. This rejection step ensures the feasibility of the individuals of the population given that the constraints were defined specifically for that purpose. The size of the population $N_{P}$ is considered as a control variable of the DE method. In this case, $N_{P}=10 m_{v}$ is used according to [34].

5.3. Mutation. The mutation allows generating a donor vector $V_{i, G_{e}}$ by means of a differential operation. This operation is defined as

$$
V_{i, G_{e}}=X_{r_{1}, G_{e}} \operatorname{OR}\left(X_{r_{2}, G_{e}} \operatorname{XOR} X_{r_{3}, G_{e}}\right),
$$

where $X_{r_{1}, G_{e}}, X_{r_{2}, G_{e}}$, and $X_{r_{3}, G_{e}}$ are three individuals of the population generation $G_{e}$ and the indices $r_{1}, r_{2}$, and $r_{3}$ are randomly selected from the range $\left[1, N_{P}\right]$. These indices must be mutually exclusive to diversify the results of the mutation operation.

The differential operation consists of adding the difference of two vectors to another vector of the population [33]. In this case, the differential operation is implemented with the exclusive disjunction (XOR). By using the logic operator $\mathrm{XOR}$, the algorithm identifies the differences between two vectors. After that, the sum operation is implemented using the disjunction operator (OR), which permits combining the difference between the two vectors with another vector of the population.

5.4. Crossover. The crossover operation allows the exchange of components between the donor vector $V_{i, G_{e}}$ and the target vector $X_{i, G_{e}}$. The result of the crossover is a trial vector $U_{i, G_{e}}=$ $\left[u_{1, i, G_{e}}, u_{2, i, G_{e}}, \ldots, u_{m_{v}, i, G_{e}}\right]$. This operation is defined as

$$
\begin{array}{ll}
u_{j, i, G_{e}}=v_{j, i, G_{e}} & \text { for } n_{v}+1 \leq j \leq n_{v}+L-1, \\
u_{j, i, G_{e}}=x_{j, i, G_{e}} & \text { otherwise, }
\end{array}
$$

where $n_{v}$ is randomly selected among $\left[1, m_{v}\right]$ with uniform probability and $L$ is obtained from $\left[1, m_{v}\right]$ with probability equal to $C_{r} \in[0,1]$. The variable $C_{r} \in[0,1]$ is known as the crossover rate and constitutes a control variable of the $\mathrm{DE}$ method. $C_{r}$ must be considerably lower than one (e.g., 0.3). If the convergence towards a solution is not achieved, then $C_{r}$ can be chosen in the interval $[0.5,1]$ according to [34].

5.5. Selection. The selection operation allows choosing between the target vector $X_{i, G_{e}}$ and the vector $U_{i, G_{e}}$ (obtained in the crossover operation) to be part of the next generation $G_{e}+1$. The selection operation is defined as

$$
\begin{array}{ll}
X_{i, G_{e}+1}=U_{i, G_{e}} & \text { if } f\left(U_{i, G_{e}}\right) \leq f\left(X_{i, G_{e}}\right), \\
X_{i, G_{e}+1}=X_{i, G_{e}} \quad \text { otherwise, }
\end{array}
$$

where $f(\cdot)$ is the objective function presented in (25) to be minimized. This operation indicates that if the trial vector $U_{i, G_{e}}$ has a value $f(\cdot)$ equal to or lower than the value $f(\cdot)$ of the target vector; then the trial vector will be part of the next generation; otherwise, the target vector $X_{i, G_{e}}$ is retained in the population of generation $G_{e}+1$. Therefore, the steps of mutation, crossover, and selection are cycled until the maximum generation $G_{\max }$ is reached. A summary of this process is presented in Algorithm 1.

5.6. Genetic Algorithm Benchmark. For the analysis of the performance of the DE algorithm, a standard Genetic Algorithm (GA) was used as a benchmark. The performance was analyzed in terms of convergence and computational cost. For the GA formulation, the chromosomes that describe each individual are binary strings, equal to those used in the DE.

The GA used has the typical stages of an evolutionary algorithm. The first stage is the generation of the initial population with the population size $\left(P_{G}\right)$ as a parameter of adjustment. Population size was chosen as 10 times the quantity of chromosome's genes $\left(N_{G}\right)$. The second stage is the selection by tournament: in this stage two individuals with the highest fitness are chosen from a randomly selected subpopulation. The third stage is crossover. A two-point crossover operation with a crossover probability of $P_{c}=0.8$ was performed. The fourth stage is mutation, which is intended to maintain a portion of random search. Mutation provides the possibility of changing one of the genes of the chromosomes of the descendants. Mutation probability was chosen as $P_{m}=0.01$. Finally, if the ending criterion (i.e., generation is equal to maximum generation, $G=G_{\max }$ ) is not reached, then a generational change is performed. In the generational change, the descendants obtained through the last stages replace their parents by initiating a new cycle. On the other hand, if the ending criterion is reached, the GA finishes. Figure 4 presents a block diagram of the GA used as a benchmark.

\section{Case Study}

The case study consists of an airport shuttle service using a $\mathrm{BEV}$ fleet. For this service, it is required to determine the route assignment and the charge scheduling in a coordinated manner. The location defined for this case study is El Dorado airport in Bogotá, Colombia. In this section, the case study is described in detail through four aspects: (i) the operation zone; (ii) the BEVs' characteristics and data logging; (iii) the data processing; and (iv) the demand for transportation.

6.1. Operation Zone. The operation zone used for this case study is defined by three nodes: El Dorado airport, defined as the north-western node; a mall located in the south node; and a public recharge station (RS) in the eastern node (see Figure 5). Within the area defined by the nodes, a total of 54 


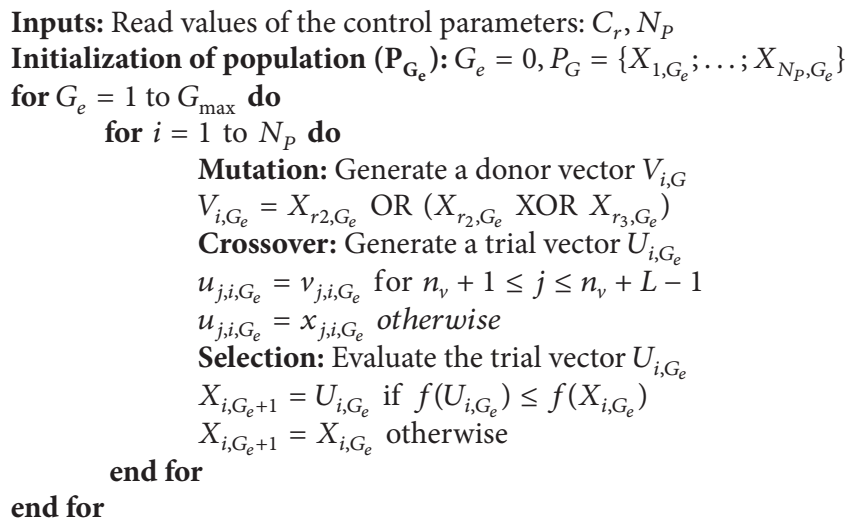

Algorithm 1: DE algorithm.

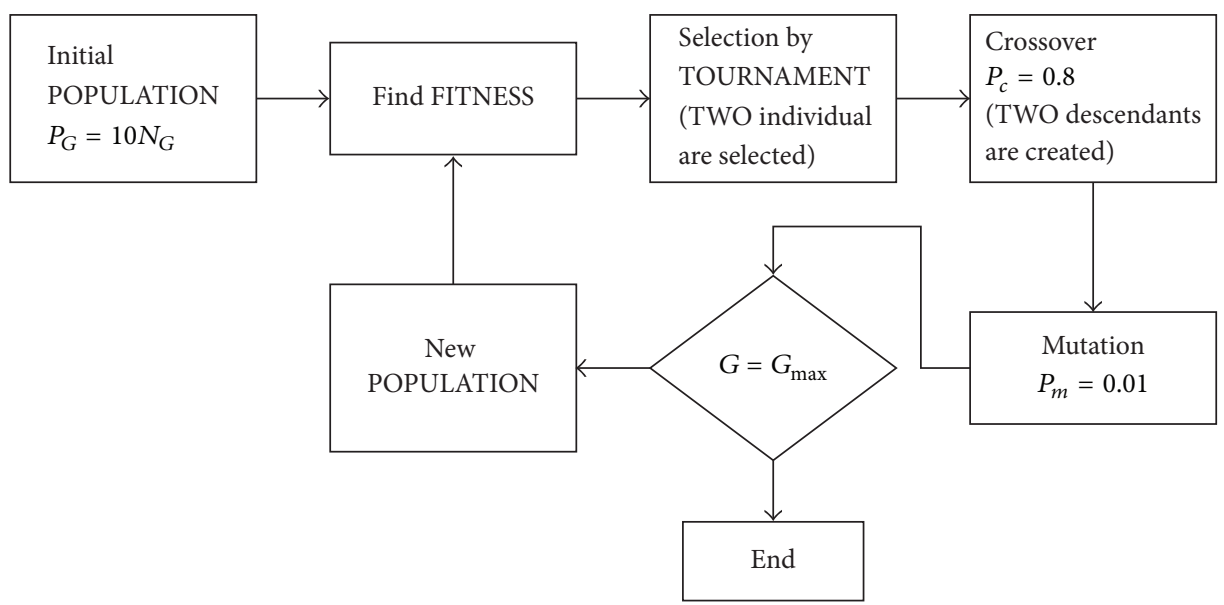

FIGURE 4: Block diagram of the GA used as a benchmark.

nodes are used as references to determine the routes. From these nodes, six are identified as main nodes and are used to build the graphics (see Figure 5 and Table 1). The main nodes are (1) airport whereabouts 1 ; (2) airport whereabouts 2 ; (3) hotel, operation center (OC), private RS with $r_{2}=3 \mathrm{~kW}, \eta_{2}=$ 0.9 ; (4) public RS with $r_{3}=6 \mathrm{~kW}, \eta_{3}=0.9$; (5) mall; and (6) bus terminal.

6.2. Test Vehicle Characteristics. The aim of the case study is to analyze the feasibility of the implementation of a fleet of $\mathrm{BEV}$ s for an airport shuttle service. In order to do this, the results of scheduling and routing are used.

The kinematical information about the displacements is needed to estimate the energy consumption of each path. This information is measured by using a different vehicle that has been selected to be as similar as possible to the BEV under study. The characteristics of the vehicle used in the case study are summarized in Table 2.

The most relevant characteristics considered to estimate the energy consumption properly are those related to resistive forces and traction acting on the vehicle. The characteristics include the dimensions (i.e., frontal area and drag coefficient) and weight to power relation of the vehicle. The test vehicle selected is a saloon type. It has been necessary to add weight to the vehicle to obtain a similar weight to power ratio.

6.3. Data Logging and Data Processing. The characteristics of the GPS unit used to measure and register the data are presented in Table 3. With the data obtained from the speed profile (see Figure 6), it has been determined that a signal conditioning stage must be implemented to handle signal noise and atypical points due to any interruptions of communication between GPS and satellites. These issues are originated by the buildings located near the streets and by bridges on the paths. Figure 6 presents an example of a lost-of-signal issue, which generates a signal spike on the raw speed profile.

The data processing considers the use of filters to deal with the data logging issues. Two filtering methods were considered to obtain a smooth speed profile: the Kalman filter described in [35] and the Savitzky-Golay filter described in $[36,37]$. Figure 7 presents a comparison between the processed data obtained by using these filters. The result obtained by using each filter is compared with the original data. The goal is to remove the signal spikes originated by 


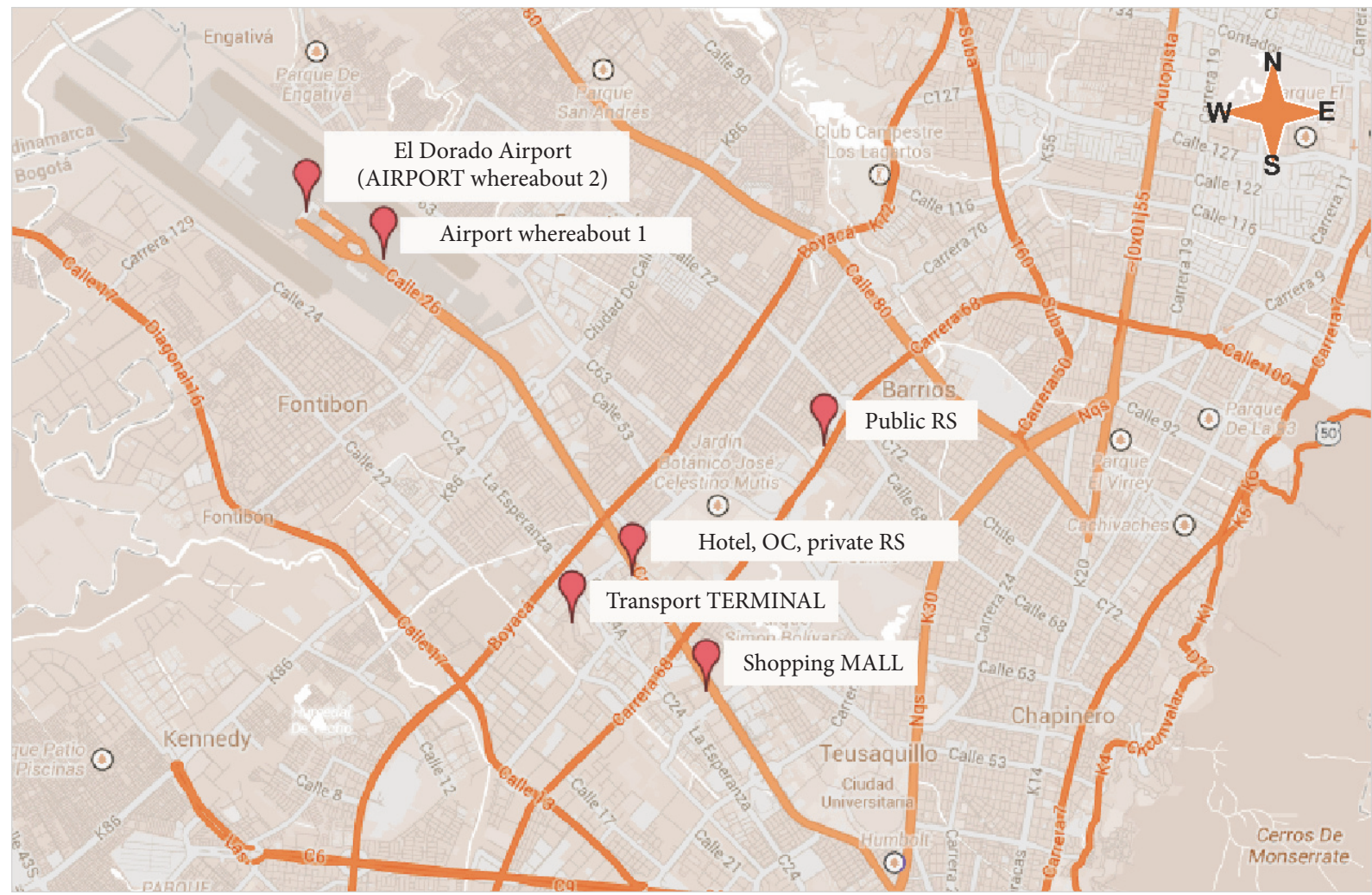

FIGURE 5: Main nodes of reference located in the operation zone.

TABLE 1: Distance matrix

\begin{tabular}{|c|c|c|c|c|c|c|}
\hline \multirow[b]{2}{*}{ Depart \arrive } & \multicolumn{6}{|c|}{ Distance $[\mathrm{km}]$} \\
\hline & Airport 1 & Airport 2 & Hotel & Public RS & Mall & Terminal \\
\hline Airport 1 & - & 0.94 & 5.92 & 9.55 & 7.62 & 6.69 \\
\hline Airport 2 & 2.94 & - & 5.86 & 9.54 & 7.6 & 6.66 \\
\hline Hotel & 6.2 & 7.11 & - & 5.5 & 1.79 & 3.33 \\
\hline Public RS & 6.99 & 7.87 & 4.21 & - & 4.21 & 5.9 \\
\hline Mall & 7.74 & 8.62 & 2.61 & 4.9 & - & 4.59 \\
\hline Terminal & 7.48 & 8.37 & 2.1 & 6.03 & 3.25 & - \\
\hline
\end{tabular}

TABLE 2: Characteristics of the test vehicle.

\begin{tabular}{lc}
\hline Parameter & Value \\
\hline$m[\mathrm{~kg}]$ & 1312 \\
$A\left[\mathrm{~m}^{2}\right]$ & 1.86 \\
$C_{D}$ & 0.32 \\
$f_{r}$ & 0.0117 \\
$\widehat{\eta}$ & 0.9 \\
Length $[\mathrm{m}]$ & 4.32 \\
Width $[\mathrm{m}]$ & 1.69 \\
\hline
\end{tabular}

the issues of the data logging process, keeping the rest of the signal unaltered. It can be observed that, for this case study, the Savitzky-Golay filter (Figure $7(\mathrm{a})$ ) is more effective than the Kalman filter (Figure 7(b)).
TABLE 3: Characteristics of the GPS unit

\begin{tabular}{lc}
\hline Parameter & Value \\
\hline Sampling frequency $[\mathrm{Hz}]$ & 100 \\
Speed resolution $[\mathrm{km} / \mathrm{h}]$ & 0.01 \\
Speed accuracy $[\mathrm{km} / \mathrm{h}]$ & 0.1 \\
Distance resolution $[\mathrm{m}]$ & 0.01 \\
Distance accuracy $[\%]$ & 0.05 \\
\hline
\end{tabular}

6.4. Transport Demand. An illustrative transport demand is defined. It consists of a case where one passenger is departing from the airport whereabouts 1 and two passengers departing from the airport whereabouts 2. This scenario is repeated every hour from 8:00 to 16:00. Each passenger must pay a 5 USD ticket and has to be picked up within a time window of 


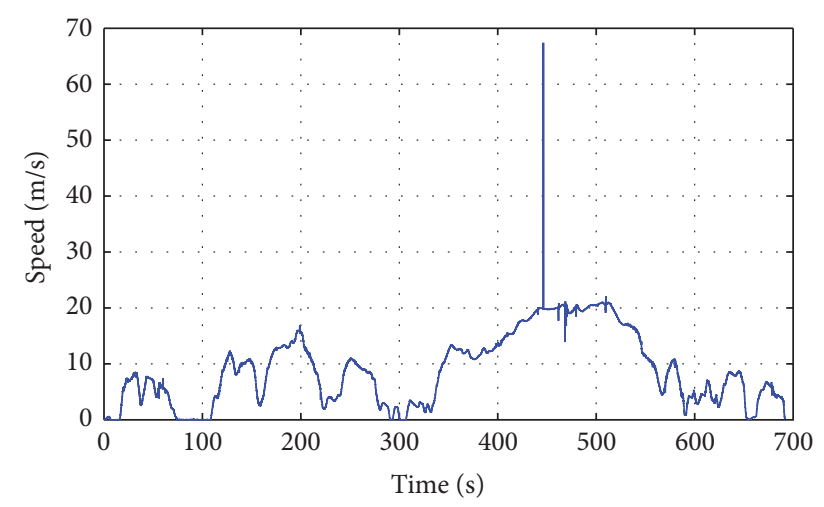

Figure 6: Raw speed profile data.

30 minutes. It is assumed that the passengers may be traveling to the mall, the terminal, or the hotel according to their needs.

6.5. Energy Consumption. The results of energy consumed by the vehicle during a trip along each path are calculated; this is achieved by the introduction of the filtered speed profile (see Figure 7) in the longitudinal dynamics model described in Section 2.1. The results are presented in the energy matrix shown in Table 4.

6.6. Simulation Results. This section presents the results of the implementation of the proposed methodology for the solution of the case study. The comparison of the proposed DE metaheuristic with a Genetic Algorithm (GA) is also presented.

The routing problem proposed is solved using the optimization tool XPRESS. The results show that nine routes must be used to satisfy the total transport demand of the case study. Each route is optimal and has an energy consumption of approximately $1.66 \mathrm{kWh}$. Traveling along each route takes less than one hour. The routes must be followed on an hourly basis during the period from 07:30 to $15: 30$ according to the transportation demand. The charge and route assignment actions are planned with a 24-hour programming horizon starting at 7:00. The programming horizon is discretized in half hour intervals. Four scenarios (SC) are considered with different working conditions to illustrate possible circumstances.

In SC1 the charge is allowed only in the private station (RS2) with a two-level energy rate: $0.20 \mathrm{USD} / \mathrm{kWh}$ between [7:00-22:00) and 0.15 USD/kWh between [22:00-7:00). For SC1 the battery degradation cost is not considered. In SC2 the charge is also allowed only in the private station (RS2) with the same two levels energy rate of SC1, and the battery degradation cost is considered.

In SC 3, the charge is allowed in both the private station (RS2) and the public station (RS3). In SC3, the energy rate of RS2 is the same one used in SC1 and RS3 has a twolevel energy rate: $0.10 \mathrm{USD} / \mathrm{kWh}$ between [7:00-22:00) and $0.05 \mathrm{USD} / \mathrm{kWh}$ between [22:00-7:00). In SC3, the battery degradation cost is considered, and the $\mathrm{V} 2 \mathrm{~B}$ operation is allowed (i.e., energy discharge of BEVs towards the hotel). In SC4, the conditions are similar to those in SC3 except for the difference in energy rate, where both RS2 and RS3 have single-level energy rates: $0.50 \mathrm{USD} / \mathrm{kWh}$ for RS2 and $0.05 \mathrm{USD} / \mathrm{kWh}$ for RS3. In SC4, the energy rate for the private station is artificially incremented to investigate a scenario where V2B operations are intuitively profitable.

The results obtained with the scenarios previously described are presented in Figures 8 and 9. For each scenario, the rate evolution and the SOC for two vehicles of the fleet and their charge actions on each station are presented across the programming horizon.

Figure 8(a) presents the charge and route assignment obtained for SC1 using DE. It is observed that the charge actions were programmed at the end of the horizon, taking advantage of the low energy rate during the night.

Figure 8 (b) presents the charge and route assignment obtained for SC2 using DE. It is found that the SOC of the BEVs describes a travel-charge pattern, meaning that the BEVs travel along one or two routes and immediately have to charge. When analyzing this behavior, it is found that the travel-charge pattern reduces the battery degradation cost by maintaining a low DOD. This is consistent with the battery model since the cycles with a large DOD are the main source of battery degradation.

SC1 and SC2 were used to compare the impact of scenario conditions on battery lifespan. When the charge and road assignments obtained for SC1 are implemented, the expected battery lifespan is approximately 6400 cycles. On the other hand, when the SC2 assignments are used, the expected battery lifespan is approximately 8400 cycles. This represents a difference of 2000 cycles, which is comparable with five years of operation of a BEV in transportation service.

Figure 9(a) presents the charge and route scheduling for SC3 obtained with DE. It was obtained that the algorithm does not schedule the V2B operation although the energy discharges are allowed. It is observed that the energy rate for SC3 is not profitable when performing the energy discharge actions because the economic benefit to afford a V2B operation is surpassed by the battery degradation cost.

Figure 9(b) presents the charge and route scheduling for SC4 obtained with DE. It was obtained that several charge actions in RS3 are programmed as well as the operation V2B through discharge actions. This behavior can be explained due to the high difference in the energy rate in the recharge stations RS2 and RS3. For this reason, the V2B operation becomes profitable. However, this difference in rates is not easily accomplished in real scenarios.

6.7. Benchmark Results. To analyze the convergence of the solution and the computational cost of the DE algorithm, a standard GA algorithm was used as a benchmark, as described in Section 5.6. The algorithms were compared under the conditions of scenarios SC1 and SC2. Given the fact that both algorithms depend on pseudorandom variables, several iterations were considered. The results are presented indicating the average values and the standard deviation obtained for each observed variable.

Table 5 presents the results obtained for the comparison under SC1 conditions. Both DE and GA converged to the same value of the daily recharge cost of a vehicle (i.e., 1.42 USD). The recharge schedules obtained were essentially 


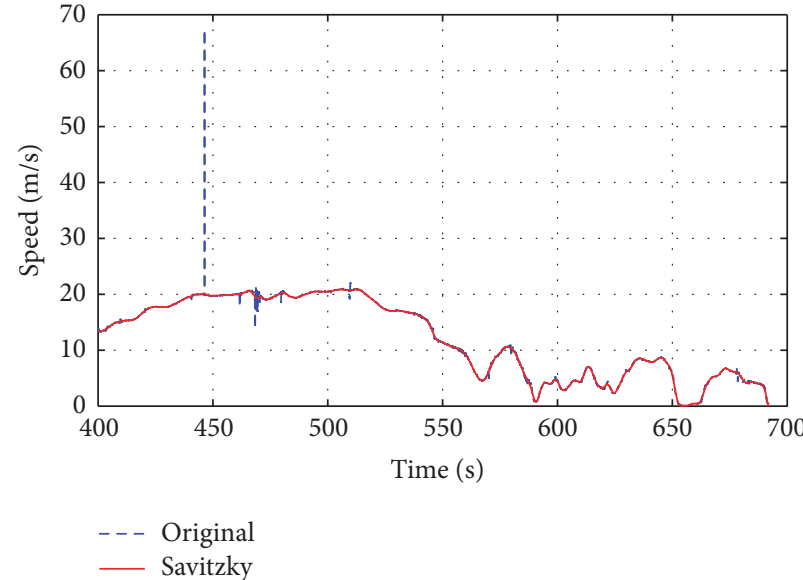

(a)

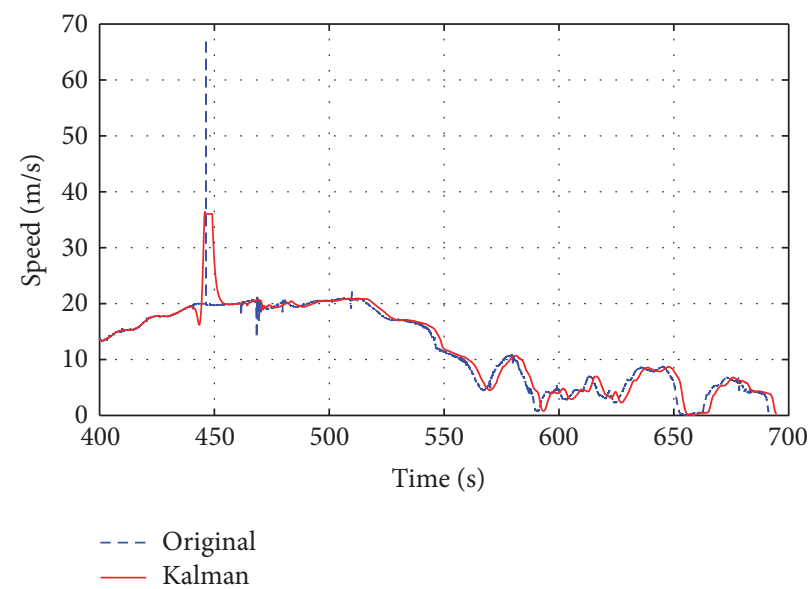

(b)

Figure 7: Comparison between filtered data. Savitzky-Golay filter (a). Kalman filter (b).
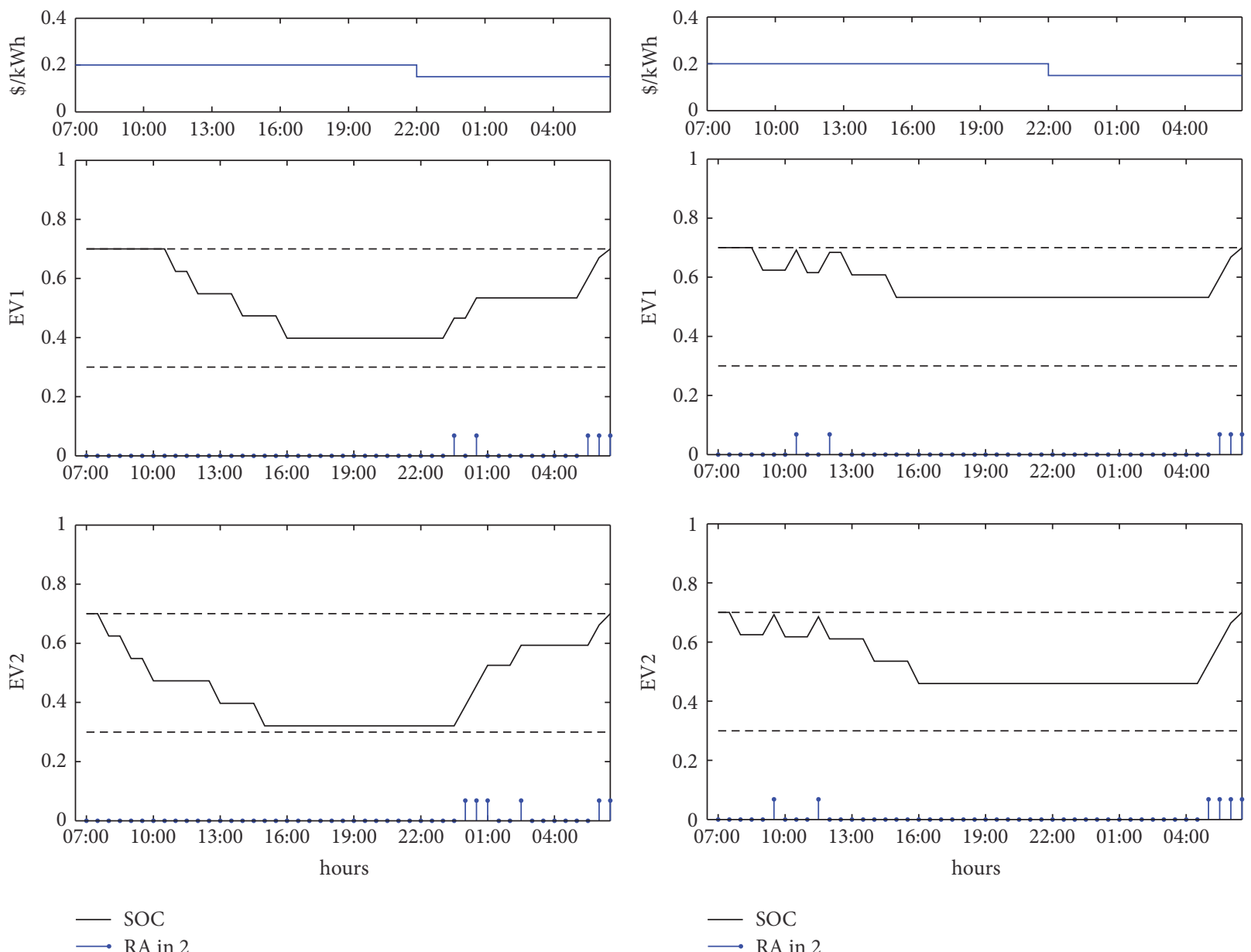

(a)

(b)

FIGURE 8: Charge scheduling for two BEVs operating in SC 1 (a) and SC2 (b). 

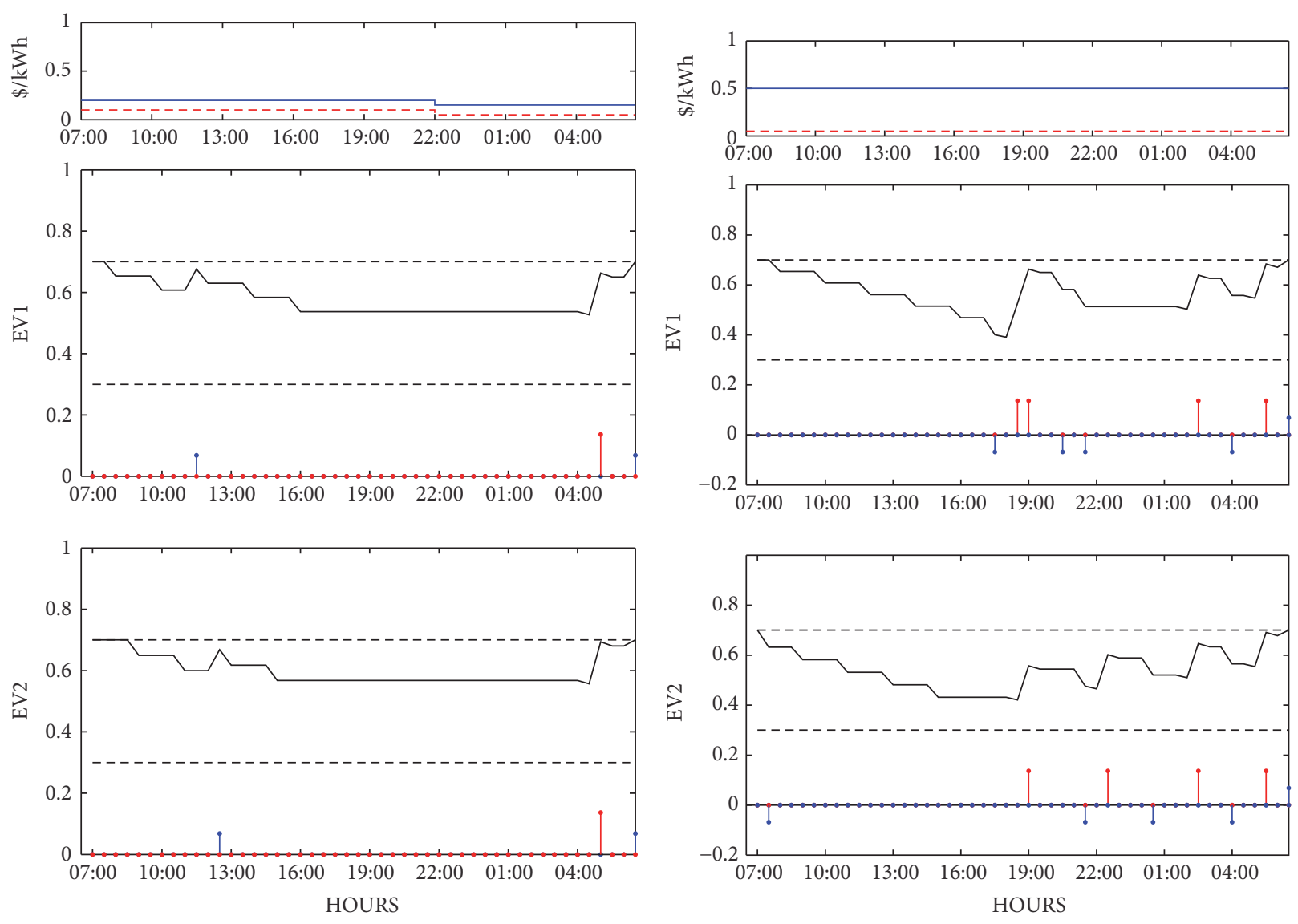

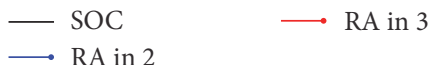

(a)

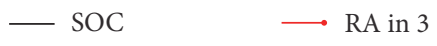

(b)

FIGURE 9: Charge scheduling for two BEVs operating in SC3 (a) and SC4 (b).

TABLE 4: Energy consumption matrix.

\begin{tabular}{lcccccc}
\hline & \multicolumn{5}{c}{ Energy consumption [kWh] } \\
Depart $\backslash$ arrive & Airport 1 & Airport 2 & Hotel & Public RS & Mall & Terminal \\
\hline Airport 1 & - & 0.128 & 0.574 & 1.073 & 0.79 & 0.719 \\
Airport 2 & 0.285 & - & 0.567 & 1.076 & 0.634 & 0.656 \\
Hotel & 0.593 & 0.773 & - & 0.658 & 0.183 & 0.429 \\
Public RS & 0.688 & 0.828 & 0.51 & - & 0.443 & - \\
Mall & 0.805 & 0.876 & 0.321 & 0.797 & 0.407 \\
Terminal & 0.839 & 0.925 & 0.28 & - \\
\hline
\end{tabular}

the same. Consequently, the recharging schedules produce a similar battery lifespan for both cases: 6434 cycles for DE and 6322 cycles for GA. Regarding the computational cost, the simulation times obtained were 14.57 for the DE and 17.91 for the GA. For SC1, the use of DE instead of GA led to a reduction of $18.6 \%$ in the computational cost. The computational cost difference obtained can be important when a large fleet is considered.

Figure 10 shows the convergence curves obtained with DE and GA. Both algorithms presented expected behaviors. The DE convergence curve is monotonously decreasing. The GA convergence curve presents some oscillations before converging. The advantage of DE is the selection process performed between generations. The choice of GA as benchmark takes advantage of its capability to reach global solutions. From this point of view, the results of the benchmark performed suggest that the DE is converging to the global minimum.

Table 6 presents the results obtained for the comparison under SC2 conditions. For this scenario, the total daily cost was computed. The total cost considers the recharging cost and the battery degradation cost. The difference between the average convergence values obtained with DE and GA for the 


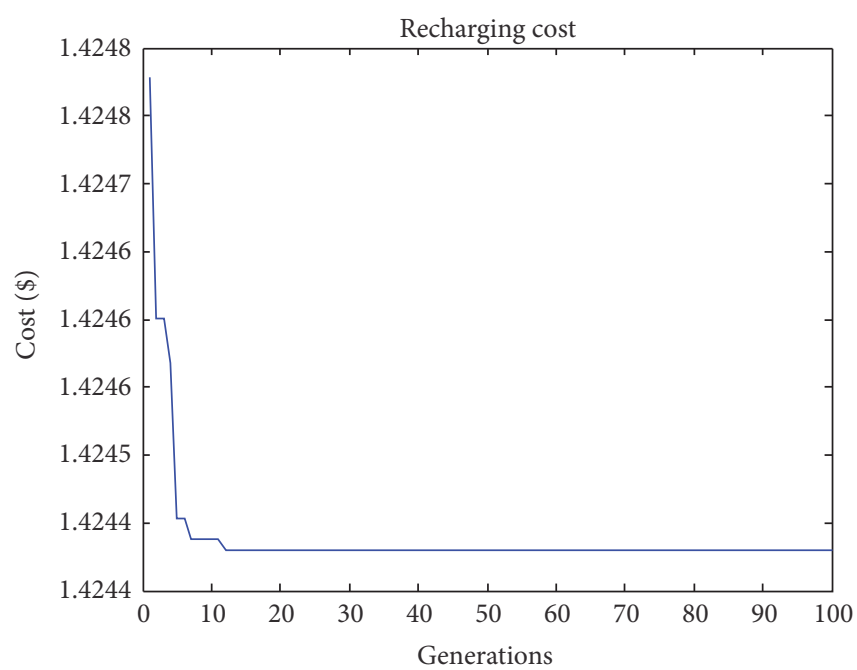

(a)

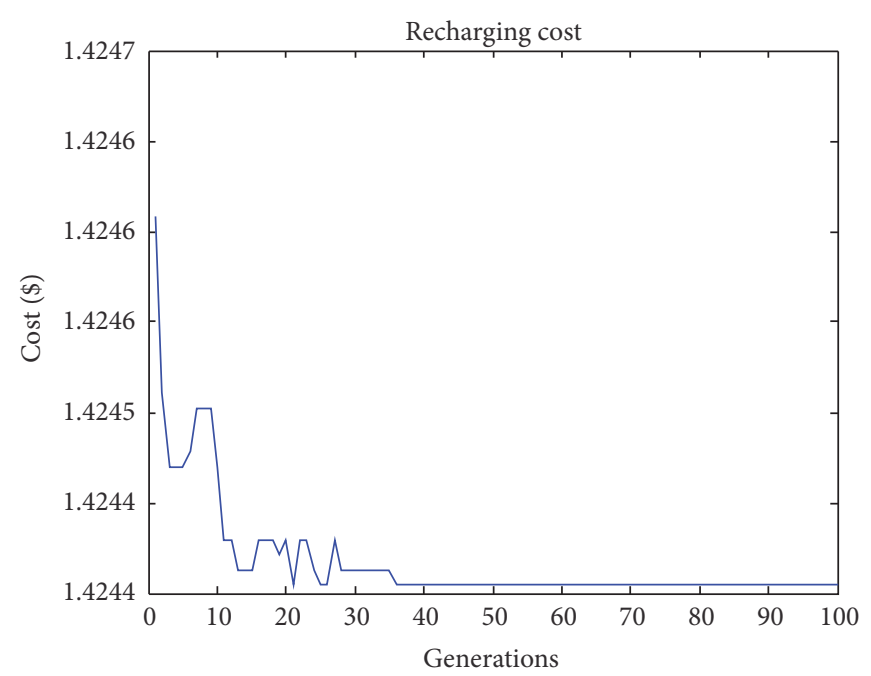

(b)

FIGURE 10: Convergence curves of DE (a) and GA (b) in SC1.

TABLE 5: Results of the simulation obtained with the DE and GA algorithms in SC1.

\begin{tabular}{lcc}
\hline & \multicolumn{2}{c}{ Average (Std. Dev) } \\
& $\mathrm{DE}$ & $\mathrm{GA}$ \\
\hline Recharge cost [USD] & $1.42(\S)$ & $1.42(\S)$ \\
Battery lifespan [cycles] & $6434(16)$ & $6322(137)$ \\
Simulation time [s] & $14.57(0.201)$ & $17.91(0.126)$ \\
\hline
\end{tabular}

$\S$ represents a standard deviation under 0.01 USD.

TABLE 6: Results of the simulation obtained with the DE and GA algorithms in SC2.

\section{Average (Std. Dev)}

$\mathrm{DE}$

\begin{tabular}{lcc}
\hline $\begin{array}{l}\text { Total cost [USD] } \\
\text { (recharging + battery } \\
\text { degradation) }\end{array}$ & $5.21(0.05)$ & $5.29(0.07)$ \\
Battery lifespan [cycles] & $8368(185)$ & $8042(281)$ \\
Simulation time [s] & $15.13(0.23)$ & $17.42(0.357)$ \\
\hline
\end{tabular}

total cost is $1.4 \%$. The difference between the average convergence values obtained with DE and GA for the battery lifespan is $3.9 \%$.

\section{Conclusions and Future Research}

A methodology to plan the energy charge and route assignation for a fleet of BEVs providing a passenger transport service has been presented. This methodology considers the search of optimal routes and the minimization of operational costs. It has been found that the charge scheduling and route assignment have effects on the battery lifetime. The result obtained for the charge scheduling allows increasing the battery lifespan. Additionally, it is observed that the consideration of battery degradation modifies the charge patterns.
In this work, some conditions to perform a V2B operation have been reported. These conditions are related to the battery technology, the battery degradation model, and the energy rate. It was found that batteries with lithium-ion technology studied in this work do not meet the requirements to provide the $\mathrm{V} 2 \mathrm{~B}$ operation as their degradation cost is high. Therefore, the V2B operation is profitable only for the $\mathrm{BEV}$ owner when the difference in energy rate between the recharge station and the discharge station is approximately $0.5 \mathrm{USD} / \mathrm{kW}$. Nonetheless, this difference in energy rates is difficult to achieve in a real scenario.

For future work, both models for the estimation of the energy consumption and for battery degradation should be refined. The energy consumption model should consider regenerative braking. The battery degradation model should consider the detailed behavior of different types of batteries. Also, the performance of additional metaheuristics should be investigated.

\section{Notations}

\section{Notation Used in the BEV Routing Problem}

$\mathscr{G}_{s}: \quad$ Simplified energy graph

$\mathscr{V}_{s}: \quad$ Vertices or nodes of the energy graph $\mathscr{G}_{s}$

$\mathscr{E}_{s}: \quad$ Edges of the energy graph $\mathscr{G}_{s}$

$v_{d_{1}}, v_{d_{2}}:$ Depot nodes

$C: \quad$ Set of customer nodes

$R_{s}: \quad$ Set of nodes on recharge stations

$P: \quad$ Set of pick-up nodes

$D: \quad$ Set of drop-off nodes

$c_{i j}$ : $\quad$ Energy consumed by traveling from node $i$

to node $j$

$t_{i j}$ : $\quad$ Time elapsed during the trip from node $i$ to node $j$

$\left[a_{i}, b_{i}\right]$ : Time window to pick up a passenger at node $i$ 
$q_{i}: \quad$ Number of passengers at node $i$

Q: Maximum passenger capacity of the BEV

$B$ : Battery capacity

$M$ : Constant, higher than any value of $w_{j}^{h}$

$e_{\min }$ : Lower bound of battery level

$H$ : $\quad$ Set of routes

$x_{i j}^{h}$ : Binary flow variable to specify that route $h$ travels between nodes $i$ and $j$

$y_{j}^{h}$ : Number of passengers picked up or dropped off at node $j$ when traveling on route $h$

$w_{j}^{h}$ : Instant at which a passenger $i$ has to be picked up or dropped off along route $h$

$e_{j}^{h}$ : Remaining level of battery charge at the end of node $i$ on route $h$.

\section{Notation Used in the Description of the Charge} Scheduling Problem

$\mathcal{N}: \quad$ Programming horizon

$N$ : $\quad$ Final time slot of programming horizon

$a_{k}^{h}$ : $\quad$ Route assignation variable

$d_{h}^{k}(n)$ : Unavailability variable

$t_{h}^{s}$ : $\quad$ Starting time for a trip along route $h$

$t_{h}^{e}$ : $\quad$ Ending time for a trip along route $h$

N: $\quad$ Assignation profile

$\operatorname{SOC}^{k}(n)$ : State of charge of BEV $k$ battery at instant $n$

$\eta_{z}: \quad$ Charge efficiency of a charge station $z$

$r_{z}: \quad$ Charge rate of charge station $z$

$u_{z}^{k}(n)$ : Charge action variable of a BEV $k$ at instant $n$ in charge station $z$

$e_{h}^{k}(n): \quad$ Variable of energy consumed by BEV $k$ at instant $n$ in route $h$

$\mathbf{u}^{k}: \quad$ Charge profile of BEV $k$

$p_{z}(n)$ : $\quad$ Energy cost in charge station $z$ at instant $n$

$c_{\mathrm{deg}}^{k}: \quad$ Battery degradation cost of BEV $k$.

Notation Used in the Description of the Battery

Degradation Model

$c_{\text {bat }}: \quad$ Battery cost

$L_{\mathrm{Q} T}$ : Battery degradation due to temperature

$L_{\mathrm{Q}, \mathrm{SOC}}$ : Battery degradation due to state of charge

$L_{\text {Q,DOD }}$ : Battery degradation due to depth of discharge

$n_{h y}$ : Number of hours in a year

$l_{y}(\cdot)$ : Function of the lifespan of the battery in years

$T_{\mathrm{amb}}$ : Ambient temperature

$R_{\mathrm{th}}: \quad$ Thermal resistance

$P_{t}(\cdot)$ : Charge power

$t_{\max }: \quad$ Time available to perform a charge in hours

$t_{\mathrm{ch}}$ : Charge time in hours

$\mathrm{CF}_{\max }$ : Battery charge capacity $y_{p}: \quad$ Battery lifespan estimated in years

$N_{l}(\cdot)$ : Battery lifespan estimated in cycles.

Notation Used in the Description of the Differential Evolution Algorithm

$\mathbf{X}^{\mathrm{U}}$ : Vector containing the charge parameters or the information about the charge actions

$\mathbf{X}^{\mathbf{a}}$ : Vector containing the route assignation parameters

$X_{i, G_{e}}:$ Vector containing the parameters of subject $i$ of generation $G_{e}$

$V_{i, G_{e}}$ : Donor vector that contains the parameters of subject $i$ of generation $G_{e}$

$U_{i, G_{e}}:$ Trial vector that contains the parameters of subject $i$ of generation $G_{e}$

$N_{p}$ : Number of parameters of the vector

$C_{r}:$ Crossover rate

$m_{v}$ : Number of elements in a parameter vector.

\section{Conflicts of Interest}

The authors declare that they have no conflicts of interest.

\section{Acknowledgments}

This work was partially supported by three sources: Project CIFI 2012, Facultad de Ingeniería Universidad de Los Andes; Codensa S.A. ESP through Silice III project, under Grant P12.245422.006.2012; and the Colombian Administrative Department of Science, Technology, and Innovation (Colciencias), Asoingeniería Ltda., and Universidad de Los Andes, under Grant 256-2013.

\section{References}

[1] A. Emadi, “Transportation 2.0," in IEEE Power and Energy Magazine, vol. 9, pp. 18-29, IEEE, 2011.

[2] C. Ozkurt, F. Camci, V. Atamuradov, and C. Odorry, "Integration of sampling based battery state of health estimation method in electric vehicles," Applied Energy, vol. 175, pp. 356-367, 2016.

[3] S. Vazquez, S. M. Lukic, E. Galvan, L. G. Franquelo, and J. M. Carrasco, "Energy storage systems for transport and grid applications," IEEE Transactions on Industrial Electronics, vol. 57, no. 12, pp. 3881-3895, 2010.

[4] A. Millner, "Modeling lithium ion battery degradation in electric vehicles," in Proceedings of the 2010 IEEE Conference on Innovative Technologies for an Efficient and Reliable Electricity Supply, CITRES 2010, pp. 349-356, Waltham, MA, USA, September 2010.

[5] L. Gaines and R. Cuenca, "Cost of Lithium-Ion Batteries for Vehicles," Center for Transportation Research, Energy Systems Division, Center for Transportation Research, Energy Systems Division, 2000.

[6] M. Sachenbacher, M. Leucker, A. Artmeier, and J. Haselmayr, "Efficient Energy-Optimal Routing for Electric Vehicles," in Proceedings of theTwenty-Fifth AAAI Conference on Artificial Intelligence, pp. 1402-1407, 2011. 
[7] N. Touati-Moungla and V. Jost, "Combinatorial optimization for electric vehicles management," Journal of Energy and Power Engineering, vol. 6, pp. 738-743, 2012.

[8] E. W. Dijkstra, "A note on two problems in connexion with graphs," Numerische Mathematik, vol. 1, pp. 269-271, 1959.

[9] D. B. Johnson, "Efficient algorithms for shortest paths in sparse networks," Journal of the ACM, vol. 24, no. 1, pp. 1-13, 1977.

[10] A. Artmeier, J. Haselmayr, M. Leucker, and M. Sachenbacher, "The Optimal Routing Problem in the Context of Battery- Powered Electric Vehicles," in proceedings of the Workshop CROCS at CPAIOR-10, Second International Workshop on Constraint Reasoning and Optimization for Computational Sustainability, pp. 1-13, 2010.

[11] S. Erdogan and E. Miller-Hooks, "A Green Vehicle Routing Problem," Transportation Research Part E: Logistics and Transportation Review, vol. 48, no. 1, pp. 100-114, 2012.

[12] D. Goeke and M. Schneider, "Routing a mixed fleet of electric and conventional vehicles," European Journal of Operational Research, vol. 245, no. 1, pp. 81-99, 2015.

[13] G. Hiermann, J. Puchinger, S. Ropke, and R. F. Hartl, "The electric fleet size and mix vehicle routing problem with time windows and recharging stations," European Journal of Operational Research, vol. 252, no. 3, pp. 995-1018, 2016.

[14] R. Roberti and M. Wen, "The Electric Traveling Salesman Problem with Time Windows," Transportation Research Part E: Logistics and Transportation Review, vol. 89, pp. 32-52, 2016.

[15] G. Desaulniers, F. Errico, S. Irnich, and M. Schneider, "Exact algorithms for electric vehicle-routing problems with time windows," Operations Research, vol. 64, no. 6, pp. 1388-1405, 2016.

[16] R. G. Conrad and M. A. Figliozzi, "The Recharging Vehicle Routing Problem," in Proceedings of the 2011 Industrial Engineering Research Conference, 2011.

[17] M. Schneider, A. Stenger, and D. Goeke, "The electric vehiclerouting problem with time windows and recharging stations," Transportation Science, vol. 48, no. 4, pp. 500-520, 2014.

[18] Z. Ma, D. Callaway, and I. Hiskens, "Decentralized charging control for large populations of plug-in electric vehicles: Application of the Nash certainty equivalence principle," in Proceedings of the 2010 IEEE International Conference on Control Applications, CCA 2010, pp. 191-195, Yokohama, Japan, September 2010.

[19] Z. Ma, D. S. Callaway, and I. A. Hiskens, "Decentralized charging control of large populations of plug-in electric vehicles," IEEE Transactions on Control Systems Technology, vol. 21, no. 1, pp. 67-78, 2013.

[20] L. Gan, U. Topcu, and S. Low, "Optimal decentralized protocol for electric vehicle charging," in Proceedings of the 2011 50th IEEE Conference on Decision and Control and European Control Conference, CDC-ECC 2011, pp. 5798-5804, Orlando, FL, USA, December 2011.

[21] L. Gan, U. Topcu, and S. H. Low, "Optimal decentralized protocol for electric vehicle charging," IEEE Transactions on Power Systems, vol. 28, no. 2, pp. 940-951, 2013.

[22] K. Clement, E. Haesen, and J. Driesen, "Coordinated charging of multiple plug-in hybrid electric vehicles in residential distribution grids," in Proceedings of the IEEE/PES Power Systems Conference and Exposition (PSCE '09), pp. 1-7, Seattle, WA, USA, March 2009.

[23] K. Clement-Nyns, E. Haesen, and J. Driesen, "The impact of charging plug-in hybrid electric vehicles on a residential distribution grid," IEEE Transactions on Power Systems, vol. 25, no. 1, pp. 371-380, 2010.

[24] E. Sortomme and M. A. El-Sharkawi, "Optimal charging strategies for unidirectional vehicle-to-grid," IEEE Transactions on Smart Grid, vol. 2, no. 1, pp. 131-138, 2011.

[25] A. Hoke, A. Brissette, D. Maksimović, A. Pratt, and K. Smith, "Electric vehicle charge optimization including effects of lithium-ion battery degradation," in Proceedings of the 7th IEEE Vehicle Power and Propulsion Conference, VPPC 2011, Chicago, IL, USA, September 2011.

[26] T. Markel, K. Smith, and A. Pesaran, "Improving Petroleum Displacement Potential of PHEV's Using Enhanced Charging Scenarios," in proceedings of the EVS-24, 24th International Battery, Hybrid and Fuel Cell Electric Vehicle Symposium, NREL/CP-540-45730, pp. 1-9, 2009.

[27] J. Hof, M. Schneider, and D. Goeke, "Solving the battery swap station location-routing problem with capacitated electric vehicles using an AVNS algorithm for vehicle-routing problems with intermediate stops," Transportation Research Part B: Methodological, vol. 97, pp. 102-112, 2017.

[28] E. S. Rigas, S. D. Ramchurn, and N. Bassiliades, "Managing Electric Vehicles in the Smart Grid Using Artificial Intelligence: A Survey," IEEE Transactions on Intelligent Transportation Systems, vol. 16, no. 4, pp. 1619-1635, 2015.

[29] L. E. Munoz, J. C. Blanco, J. P. Barreto, N. A. Rincon, and S. D. Roa, "Conceptual design of a hybrid electric off-road vehicle," in Proceedings of the 2012 IEEE International Electric Vehicle Conference, IEVC 2012, Greenville, SC, USA, March 2012.

[30] P. Toth and D. Vigo, Eds., The vehicle routing problem, vol. 9 of SIAM Monographs on Discrete Mathematics and Applications, Society for Industrial and Applied Mathematics (SIAM), Philadelphia, PA, 2002.

[31] V. Marano, S. Onori, Y. Guezennec, G. Rizzoni, and N. Madella, "Lithium-ion batteries life estimation for plug-in hybrid electric vehicles," in Proceedings of the 5th IEEE Vehicle Power and Propulsion Conference, VPPC '09, pp. 536-543, Dearborn, MI, USA, September 2009.

[32] R. Storn and K. Price, "Differential evolution: a simple and efficient heuristic for global optimization over continuous spaces," Journal of Global Optimization, vol. 11, no. 4, Article ID 341359, pp. 341-359, 1997, http://dx.doi.org/10.1023/A:1008202821328.

[33] S. Das and P. Suganthan, "Differential evolution: a survey of the state-of-the-art," IEEE Transactions on Evolutionary Computation, vol. 15, no. 1, pp. 4-31, 2011.

[34] R. Storn, "On the Usage of Differential Evolution for Function Optimization," in Proceedings of North American Fuzzy Information Processing, pp. 519-523, 1996.

[35] R. E. Kalman, "A new approach to linear filtering and prediction problems," Journal of Basic Engineering, vol. 82, no. 1, pp. 35-45, 1960.

[36] S. J. Orfanidis, Introduction to Signal Processing, Prentice Hall, 2010.

[37] A. Savitzky and M. J. E. Golay, "Smoothing and differentiation of data by simplified least squares procedures," Analytical Chemistry, vol. 36, no. 8, pp. 1627-1639, 1964. 


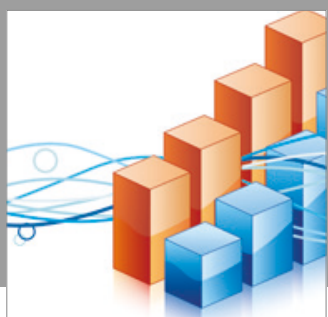

Advances in

Operations Research

vatersals

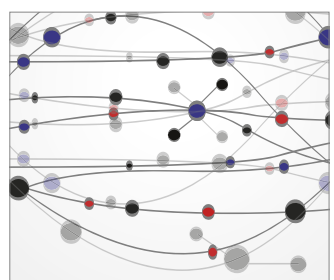

\section{The Scientific} World Journal
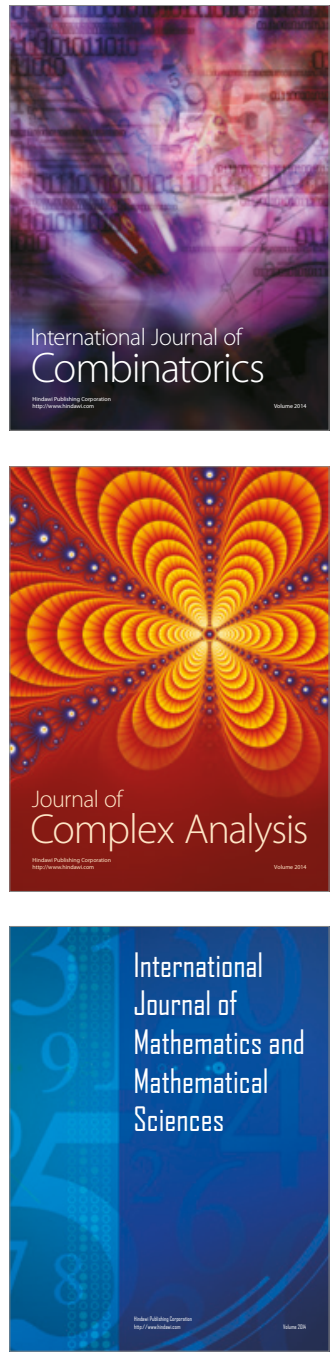
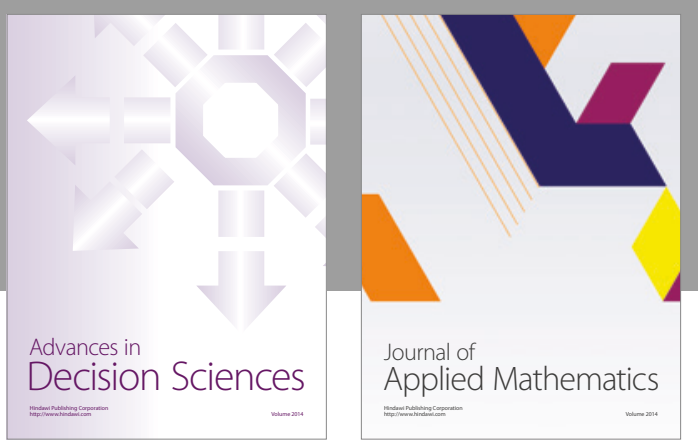

Algebra

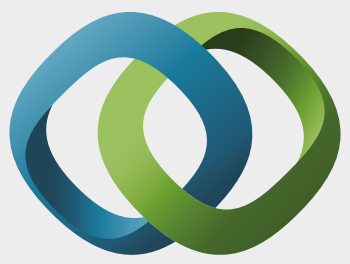

\section{Hindawi}

Submit your manuscripts at

https://www.hindawi.com
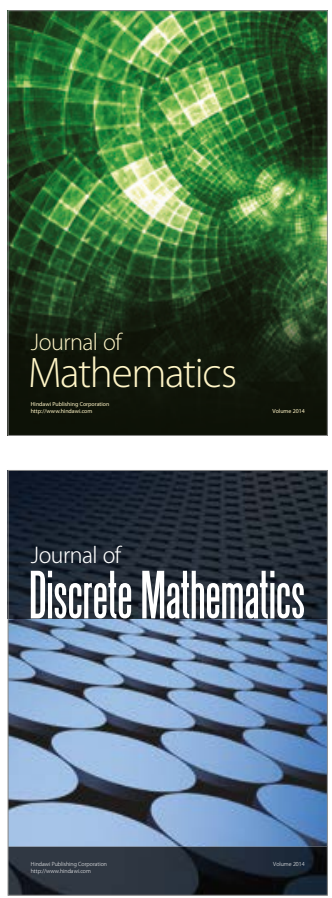

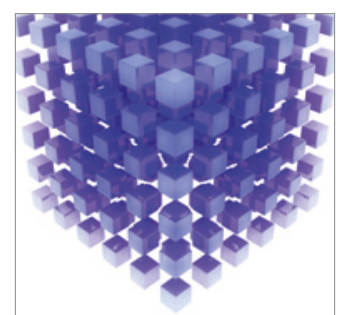

Mathematical Problems in Engineering
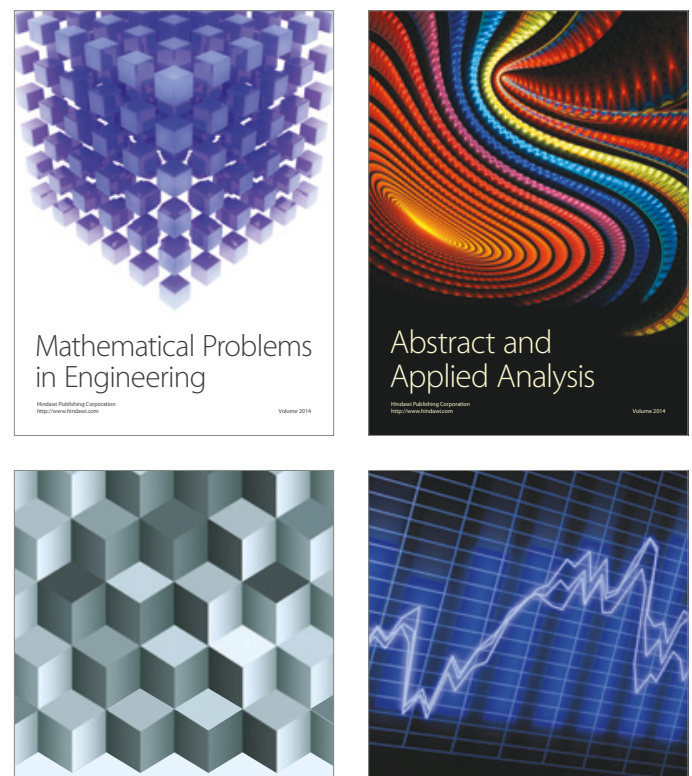

Journal of

Function Spaces

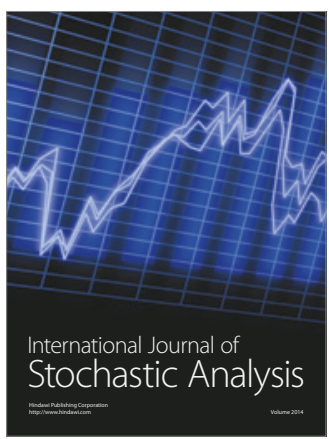

Probability and Statistics
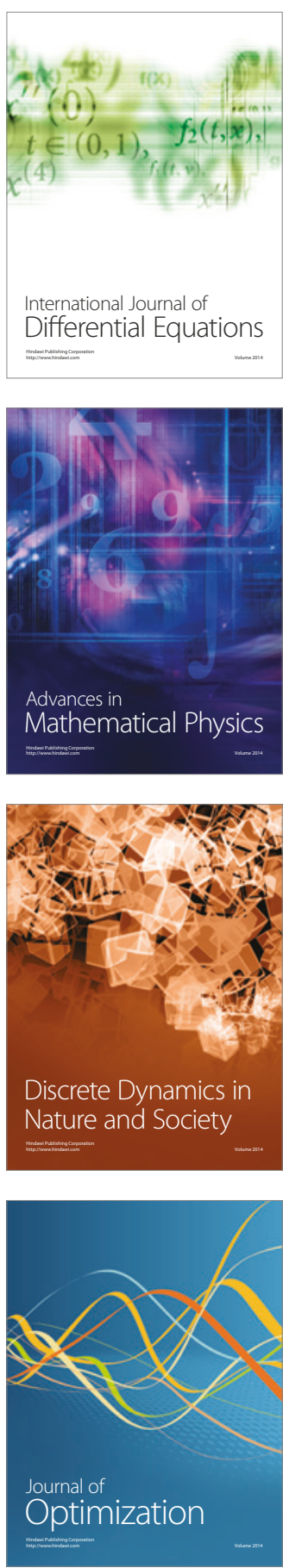